\title{
New Development of Classical Actuator Disk Model for Propellers at Incidence
}

\author{
Rafael L. Rubin* and Dan Zhao \\ University of Canterbury, Christchurch 8041, New Zealand \\ https://doi.org/10.2514/1.J059734
}

\begin{abstract}
The actuator disk model thrust formula was mathematically expanded in series and divided into two parts to show that propellers at incidence comprise an axial and a wing lift equivalent component. Both components share a common induced speed $w$. This is done by considering an enhanced disk area for momentum balance, to match Glauert's hypothesis mass flowrate. To shed light on the theoretical developments, wind tunnel tests were conducted on a two-bladed propeller at angles of incidence ranging from 0 to $90 \mathrm{deg}$. The wing component is shown to increase with airflow velocity and angle of incidence. The axial component decreases with $V$, for all angles. The generally observed thrust increase with angle of incidence is explained, by the theory, to be mostly due to the wing component contribution. The theory also explains why at around an angle of incidence of $60 \mathrm{deg}$ propellers inherently behave differently than at lower angles. While thrust decreases with $V$ at lower angles, it grows with airspeed at an angle of incidence of approximately $60 \mathrm{deg}$ or higher. This behavioral inversion happens as the wing component positive sensitivity to $V$ overcomes the negative sensitivity of the axial component. A simplified formula is presented for predicting thrust at a given angle, based only on propellers data at an angle of incidence of 0 , regardless of blade geometry.
\end{abstract}

AOA, $\alpha_{p}$
$\alpha_{\text {slp }}$
$\alpha_{\text {ult }}$
$C_{T}$
$D$
$e$
$e_{N}$
$e_{T}$
$F_{x}$
$F_{z}$
$J$
$M_{p}$
$M_{y}$
$\dot{m}$
$N_{p}$
$n$
$p$
$p_{\text {atm }}$
$q$
$R P M$
$S_{\text {disk }}$
$S_{\text {eff }}$
$S_{\text {wing }}$
$T$
$T_{\text {axial }}$
$T_{\text {wing }}$
$V$
$V$
$V_{\text {disk }}$

$\quad$ Nomenclature
$=$ propeller's angle of incidence
$=$ slip-stream angle of incidence at rotor disk
$=$ slip-stream ultimate angle of incidence
$=$ thrust coefficient, equal to $T / \rho n^{2} D^{4}$
$=$ propeller's diameter
$=$ theoretical entrainment factor
$=$ direction versor of propeller normal force due to
$=$ angle of incidence
$=$ thrust direction versor or propeller axial direction
$=$ wind tunnel net force measured on $x$ axis
$=$ advance ratio, equal to $V / n D$
$=$ propeller's pitching moment due to angle of
$=$ incidence
$=$ wind tunnel net moment measured on $y$ axis
$=$ propeller's normal force due to angle of incidence
$=$ propeller's frequency
$=$ static pressure
$=$ atmospheric pressure
$=$ freestream dynamic pressure, equal to $0.5 \rho V^{2}$
$=$ propeller's revolution per minute
$=$ propeller's swept area or rotor disk area
$=$ propeller's momentum balance effective area
$=$ equivalent wing area for $T_{\text {wing }}$ calculation
$=$ propeller's thrust
$=$ axial component of thrust
$=$ wing lift equivalent component of thrust
$=$ freestream or wind velocity
$=$ freestream velocity vector
slip-stream velocity at rotor disk

Received 7 May 2020; revision received 2 September 2020; accepted for publication 2 September 2020; published online Open Access XX epubMonth XXXX. Copyright @ 2020 by Rafael Levy Rubin and Dan Zhao. Published by the American Institute of Aeronautics and Astronautics, Inc., with permission. All requests for copying and permission to reprint should be submitted to CCC at www.copyright.com; employ the eISSN 1533-385X to initiate your request. See also AIAA Rights and Permissions www.aiaa.org/randp.

*Ph.D. Candidate, Department of Mechanical Engineering; Aerospace Engineer, Private Bag 4800. rubin.rafael@ gmail.com.

${ }^{\dagger}$ Associate Professor, Department of Mechanical Engineering, Private Bag 4800; dan.zhao@canterbury.ac.nz. Associate Fellow AIAA.

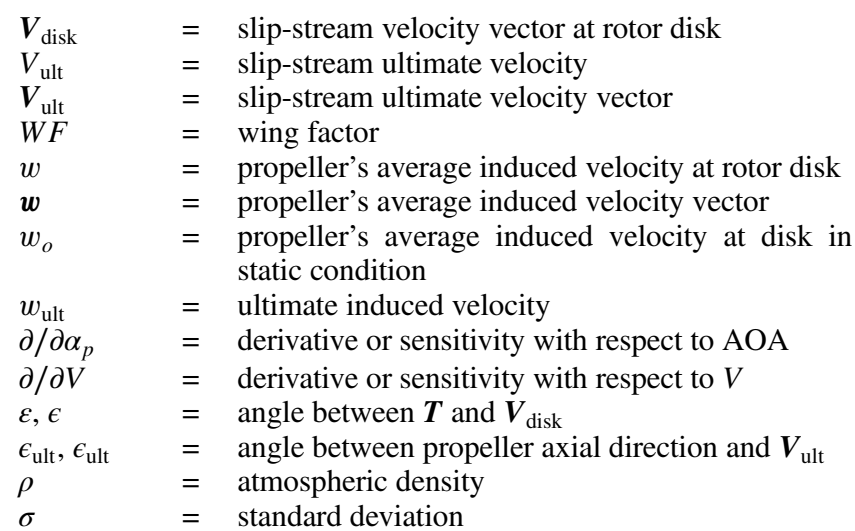

Subscripts

ult $=$ ultimate or far wake

$\left.\right|_{V=0} \quad=\quad$ static condition

$\left.\right|_{\mathrm{AOA}=0 \text { deg }}=$ no incidence condition

\section{Introduction}

$\mathbf{L}$ ARGE interest in vertical takeoff and landing (VTOL) tilt-mode aircraft development appeared in the late 1940s, requiring research on propellers performance for a wide range of angles of incidence. Recently, VTOL unmanned aerial vehicles (UAVs) projects are causing the resurgence of the subject for small scaled propellers. In most cases, propellers are designed either for near-axial flight for airplanes or to perform as helicopter rotors. The transition between these flight modes in tilt-mode vehicles is mostly brief. Nonetheless, understanding and modeling propellers behavior at all angles is crucial to determine those vehicles performance.

In the past century, several models with different accuracies and complexities were developed. The simplest model to assess propeller thrust performance is the classical momentum theory or actuator disk model. It was first introduced for marine propellers by Rankine [1] in 1865 and Froude [2] in 1889. Later, in the beginning of the 20 th century, it was adopted for airscrews with the advent of the airplane. The theory applies the basics of fluid dynamics conservation laws [mass (continuity), momentum, and energy] to provide a general understanding of the propeller performance. Its assumptions disregard the propeller blades' geometry and details of the flow about them. The propeller is considered as an infinitely thin actuator disk 
that impels a sudden increase in pressure on the fluid as it flows across its surface. This causes an acceleration of the flow, so an induced velocity increment is modeled at the disk. The flow is assumed to be incompressible and inviscid and rotation is neglected. Also, the velocity and pressure over the disk are considered to be uniform, and so thrust is distributed evenly over its surface. The major limitations of the theory are that it does not take profile drag losses of the propeller blades nor blades' tip vortices and rotation effects into account. Despite the simplifications assumed, the patterns of velocities and pressures in the actuator disk model have been verified experimentally [3] , and although it is not very accurate for power estimates [4], it provides a very good approximation for thrust. The theory is, however, not expected to yield a good basis for rotor in plane analysis, and hence it is not suitable for propeller design by itself. The derivation of the equations involved in the classical axial momentum theory for propellers can be found in several sources [5-8].

Subsequent models with higher complexity arose in the wake of the actuator disk theory. General or extended momentum theory, where the incorporation of rotation is added to the model, was developed by Betz [9]. The blade element theory, which considers the geometry of the blades, was first devised by Drzewiecki [10] in 1920. It ignored the effect of the induced flow inside the stream tube [11], as defined in the momentum theory, taking into account only the freestream velocity $V$ and the propeller rotation for every blade element analysis. The more precise blade element-momentum theory (BEMT) incorporated the induced velocity. Other variations of BEMT calculate the induced velocity in different ways. Goldstein's classical vortex theory [12] related the induced velocity to the bound circulation around every blade element. Vortex theory studies were later enhanced by Theodorsen [13]. Extensive integrations of rotor aerodynamics analysis by Joukowsky in light of momentum theories and under vortex theories are presented in Refs. [14] and [15], respectively.

Previous relevant studies to incorporate nonaxial flow conditions were made by Ribner [ $[16,17]$, who devised formulas, using BEMT, to calculate side forces and moments that appear when propellers operate at incidence. The development assumed low angles of incidence. De Young [18] later modified the Ribner formulas and expanded the use for high angles and also analyzed the effects of incidence on thrust. Experimental investigations have also been made in order to understand the behavior of full-scale propellers at a wide range of incidence in Refs. [19-21], in which it has been shown that thrust increases with increasing $\mathrm{AOA}$ and that thrust grows with the advance ratio $J$, at high $\mathrm{AOA}$, as opposed to the effect at low angles. Recently, experiments on several small scaled propellers for UAV applications $[\underline{22}, \underline{23}]$ also showed that for AOA $<60 \mathrm{deg}, C_{T}$ diminishes with the advance ratio $J$, and vice versa for higher angles of incidence. Previous studies on wind turbines under yaw conditions are reported in Refs. [24-31].

Glauert [7,32] conducted an analysis on helicopter rotors in forward flight using momentum theory. Although it is mentioned that no solid proof has been given, he introduced a thrust formula for angles of incidence that reverts to the classical static thrust equation in the case of zero forward speed, and at the other extreme at fast forward flight, the equation takes the form of the elliptic wing lift formula, implying that the rotor behaves as a wing in that case. Bramwell [33] investigated the validity of Glauert's formula by solving the linearized Euler's equations with small disturbances in the velocity field, showing that, for lightly loaded propellers, the model is valid for the axial case and for high speeds at $90 \mathrm{deg}$, regardless of blade geometry. Moreover, in the latter case, the linear theory appears to hold also for nonuniform load distributions. Glauert's hypothesis for propellers at incidence has been widely used also for a wide angle of attack (AOA) range $[\underline{3}, \underline{4}, \underline{8}, \underline{11}]$.

The appeal of the actuator disk momentum theory simplicity attracted further investigation to determine its limitations and possible improvements of its accuracy. Van Kuik $[34,35]$ mentions that the average induced velocity calculated with the assumptions of the classical theory is underestimated. For a uniform load, a singularity at the disk edge must be included through a correction represented by a discrete vortex carrying an edge force. Goorjian [36] also states an inconsistency in the general momentum theory, as mutual interferences between different annular elements are not considered. The existence of this inconsistency was also known to Glauert, but only in recent years, the errors associated with it have been quantified. This is due to Sorensen and Mikkelsen [37], Van Kuik and Lignarolo [38], and Bontempo and Manna [39] for the axial momentum theory, and in the general case, to Sorensen [40], and Bontempo and Manna [41,42]. Conway [43] developed an analytical closed solution for the linearized actuator disk with arbitrary radial load distributions. As an extension of the linearized solution, a semi-analytical method was then developed for a nonuniform heavily loaded disk in [44]. Pitt and Peters [45] presented a linear, unsteady actuator model considering the dynamic inflow behavior of helicopter rotors. A model for axial and for skewed flows by Morillo and Peters [46] presents the solution of the complete velocity field above the disk by converting the potential flow equations into ordinary differential ones. Recently, Rosen and Gur [47] developed an axisymmetric axial actuator disk model including radial and tangential induced speed components. The model defines a pressure ratio factor that depends on the blades geometry to conclude the momentum balance, for which calculations are performed iteratively. Later, Kominer and Rosen [48] adapted the model for asymmetric skewed inflows.

Although the previously cited actuator disk models improve accuracy, many of them are cumbersome to implement and end up missing the advantage of the momentum theory simplicity. For high accuracy, and alternative investigation approach for propellers at angles of incidence uses computational fluid dynamics (CFD) to solve the three-dimensional (3D) flow equations. These models may include viscosity, nonuniform propeller inflows, complex geometry one and more complicating assumptions such as the addition of nacelles and wing interferences in the analysis $[22,49,50]$. Often, high-complexity CFD models are coupled with experimental tests for validation. In general, they are also computational expensive and time consuming to implement.

In this Paper, we conduct theoretical studies by following the classical momentum theory and on Glauert's hypothesis [7] for aerodynamic propellers at incidence. By considering an enhanced mass flow rate through the disk, this new development allows for a mathematical decomposition of thrust into two parts: $T_{\text {axial }}$, which is dependent on the axial component of the oncoming wind speed, and $T_{\text {wing }}$ which is sensitive to the wind component parallel to the rotor plane. This is done in Sec. II. The development quantifies the contribution of each component to total thrust and helps clarify, under the scope of momentum theory alone, why rotors in forward flight behave as wings and why thrust increases with AOA. To validate the theoretical findings, experimental wind tunnel tests are conducted on a twoblade propeller at angles of incidence ranging from 0 to $90 \mathrm{deg}$. This is described in Sec. III. The effects of revolutions per minute, AOA, and oncoming flow velocity on thrust measurements are experimentally evaluated, and the propeller's behavior is explained, through the influence of the two components, in Sec. VI. Furthermore, as a consequence of the analysis, a simplified formula is derived to allow for the estimation of propellers thrust when at incidence, based on the performance data measured at $\mathrm{AOA}=0$. A discussion on slip-stream parameters in response to AOA is also provided. Key findings are summarized in Sec. $\underline{\text { V. }}$

\section{Momentum Theory for Propellers at Incidence}

\section{A. Theoretical Entrainment Factor $e$}

The classical momentum theory models an inviscid flow, with a uniform pressure jump and an average uniform induced speed at the actuator disk. Here, rotation is neglected. To apply the principles of momentum theory at incidence, it is first necessary to define the mass flow rate through the disk and the boundaries of the stream tube. As the airflow velocity at the disk $V_{\text {disk }}$ is not normal to the disk plane (see Figs. 1a and 1b), initially one would assume the boundaries of the stream tube touching the disk rim and the normal component of $V_{\text {disk }}$, in other words, $\left(V \cos \alpha_{p}+w\right)$, to estimate the mass flux through the disk. However, it has been determined experimentally that the rotor entrains air from outside the rim stream tube [8]. The phenomenon can be explained to be caused by radial pressure gradients effects not 


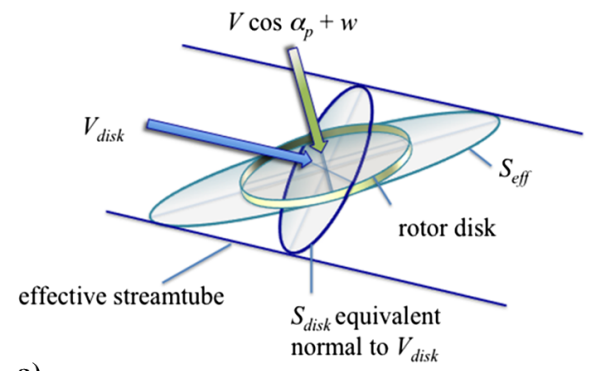

a)

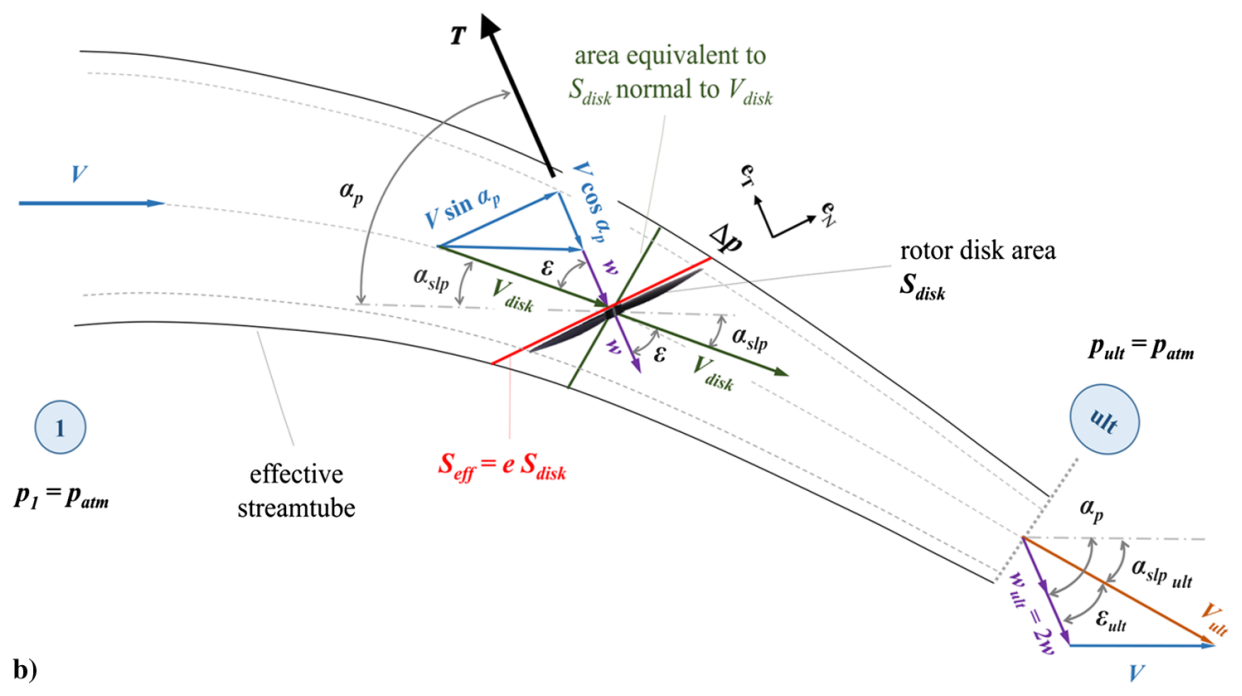

Fig. 1 Scheme of a propeller at incidence: a) effective stream tube definition and b) illustration of velocities vectors.

modeled in the classical momentum theory. The radial pressure gradients cause an increase in mass flow that grows with AOA, by means of tip vortices. A rigorous description of the phenomenon can be found in Refs. [34-37]. As the radialmomentum balance is disregarded, the uniform pressure load at the disk would cause an infinite radial pressure gradient at the edge and a velocity singularity, which are inconsistent. This inconsistency in the model can only be resolved through the addition of edge vortices, which act as natural concentrators (see Refs. [34,51]), which increase the mass flow through the disk. This effect grows with AOA and with the speed component in the rotor plane (see Ref. [48]).

To incorporate an increase in mass flow rate to the model, an entrainment factor $e$ is applied to the disk area, and a new effective area $S_{\text {eff }} S_{\text {eff }}=e S_{\text {disk }}$ is defined. Then, $\dot{m}$ can be expressed as

$$
\dot{m}=\rho\left(V \cos \alpha_{p}+w\right) S_{\text {eff }}
$$

Shapiro [3] also postulates the hypothesis of a much larger region of induced flow affected by the rotor at incidence and assumes a wider stream tube, where the mass of air that takes part in the exchange of momentum is the mass flux through a projected area normal to $V_{\text {disk }}$ and equivalent to $S_{\text {disk }}$, regardless of the angle of incidence. In other words, this wider effective stream tube can be defined by flipping the rotor disk area to a normal position relative to $V_{\text {disk }}$ (see Fig. 1). The mass flow rate in the effective stream tube would then be given as

$$
\dot{m}=\rho V_{\text {disk }} S_{\text {disk }}
$$

Now, stretching the disk area at the original position to reach the new stream tube boundaries will define a new enhanced or effective disk area $S_{\text {eff }}$ that is adopted for the mass flux calculation in Eq. (1). The following development is based on the assumption that Eqs. (1) and (2) are equivalent for the definition of the mass flowrate $\dot{m}$. This concept that determines the mass flowrate through a circle, with an area defined by the propeller diameter and normal to $V_{\text {disk }}$, comes from rotor analysis in forward flight and uses the analogy to the wing theory [3, $, 2,7,52]$. It was presented in Glauert's hypothesis [7].

The freestream velocity projected on the propeller's reference frame can be shown as

$$
V=-V \cos \alpha_{p} \boldsymbol{e}_{T}+V \sin \alpha_{p} \boldsymbol{e}_{N}
$$

By following the momentum theory, where the velocity at the disk is the sum of the axial induced velocity by the propeller and the freestream speed, $\boldsymbol{V}_{\text {disk }}=\boldsymbol{V}+\boldsymbol{w}$, then it is quite obvious that the following two relationships hold:

$$
\begin{aligned}
& \boldsymbol{V}_{\mathrm{disk}}=-\left(V \cos \alpha_{p}+w\right) \boldsymbol{e}_{T}+V \sin \alpha_{p} \boldsymbol{e}_{N} \\
& \left|V_{\mathrm{disk}}\right|=\sqrt{\left(V \cos \alpha_{p}+w\right)^{2}+\left(V \sin \alpha_{p}\right)^{2}}
\end{aligned}
$$

As illustrated in Fig. 1b, it can be determined from vector geometric relationship that

$$
V_{\text {disk }} \cos \varepsilon=\left(V \cos \alpha_{p}+w\right)
$$

Substituting Eqs. (2) and (5) into Eq. (1) leads to the theoretical entrainment factor $\bar{e}$ being determined as

$$
e=\frac{V_{\text {disk }}}{\left(V \cos \alpha_{p}+w\right)}=\sqrt{1+\frac{\left(\sin \alpha_{p}\right)^{2}}{\left(\cos \alpha_{p}+w / V\right)^{2}}}=\frac{1}{\cos \varepsilon}
$$

Hence, the effective area and the disk area are related to each other as

$$
S_{\text {eff }}=\frac{S_{\text {disk }}}{\cos \varepsilon}
$$

With $e$ and the mass flow rate predicted, now we consider the momentum balance in the propeller axis direction $\boldsymbol{e}_{T}$, between the 
ultimate wake section far downstream in the stream tube and section 1 far upstream of the propeller. Recalling that the classical momentum theory disregards the wake rotation effects and assumes the flow being inviscid, then the total thrust is the only force imparted to the flow. It can be written mathematically as

$$
T=\dot{m}\left(V_{\mathrm{ult}} \cos \varepsilon_{\mathrm{ult}}-V \cos \alpha_{p}\right)
$$

by applying geometry relationship from Fig. $1 \mathrm{~b}$ it is possible to see that $V_{\text {ult }} \cos \varepsilon_{\text {ult }}=\left(V \cos \alpha_{p}+w_{\text {ult }}\right)$, which leads to

$$
T=\dot{m} w_{\mathrm{ult}}
$$

Substituting Eq. (1) in (9) allows thrust to be rewritten as

$$
T=\rho\left(V \cos \alpha_{p}+w\right) S_{\text {eff }} w_{\text {ult }}
$$

Considering the streamlines of the stream tube between section 1 and immediately before the disk (-) and from immediately after the disk $(+)$ to the ultimate wake, the following two Bernoulli equations are obtained:

$$
\begin{gathered}
p_{1}+\frac{1}{2} \rho V^{2}=p_{\text {disk }}^{-}+\frac{1}{2} \rho V_{\text {disk }}^{-2} \\
p_{\text {ult }}+\frac{1}{2} \rho V_{\text {ult }}^{2}=p_{\text {disk }}^{+}+\frac{1}{2} \rho V_{\text {disk }}^{+2}
\end{gathered}
$$

Here, $V_{\text {disk }}^{-}=V_{\text {disk }}^{+} \triangleq V_{\text {disk }}$ for continuity through the disk, and $p_{1}=p_{\text {ult }}=p_{\text {atm }}$. This far wake pressure recovery can only be completed in the inviscid actuator disk model, as the wake rotation is neglected $[7,37,39]$. At the disk, there is a jump in pressure $\Delta p$ impelled by the propeller. Thus, $p_{\text {disk }}^{+}=p_{\text {disk }}^{-}+\Delta p$. Manipulating Eqs. (11a) and (11b) and applying the pressure jump definition leads to

$$
\frac{1}{2} \rho\left(V_{\mathrm{ult}}^{2}-V^{2}\right)=\Delta p
$$

where $V_{\text {ult }}$ as illustrated in Fig. $1 \mathrm{~b}$ is given as

$$
V_{\mathrm{ult}}^{2}=\left(\mathrm{V}+w_{\mathrm{ult}} \cos \alpha_{p}\right)^{2}+\left(w_{\mathrm{ult}} \sin \alpha_{p}\right)^{2}
$$

By substituting Eqs. (13) into (12) and considering that the force causing the jump in pressure is due to the thrust $T$, the pressure jump can be rewritten as

$$
\Delta p=\frac{T}{S_{\text {eff }}}=\frac{1}{2} \rho\left(\left(V+w_{\mathrm{ult}} \cos \alpha_{p}\right)^{2}+\left(w_{\mathrm{ult}} \sin \alpha_{p}\right)^{2}-V^{2}\right)
$$

Substituting Eq. (10) into Eq. (14) and expanding terms in the rhs leads to

$$
\begin{gathered}
\rho\left(V \cos \alpha_{p}+w\right) w_{\mathrm{ult}}=\frac{1}{2} \rho\left(w_{\mathrm{ult}}^{2}+2 w_{\mathrm{ult}} V \cos \alpha_{p}\right) \\
w_{\mathrm{ult}}=2 w
\end{gathered}
$$

The thrust $T$ can be expressed by using Eqs. (15b) and (10) as

$$
T=2 \rho\left(V \cos \alpha_{p}+w\right) e S_{\mathrm{disk}} w
$$

On the other hand, $T$ can also be rewritten from Eqs. (2), (9), and $(\underline{15 b})$ as

$$
T=2 \rho V_{\mathrm{disk}} S_{\mathrm{disk}} w
$$

Equation (17) is the well-known equation of thrust from Glauert's hypothesis [7]. Substituting Eq. (4b) into Eq. (17) leads to a quartic relationship in terms of $w$ as

$$
\left(\frac{T}{2 \rho S_{\text {disk }}}\right)^{2}=V^{2} w^{2}+2 V w^{3} \cos \alpha_{p}+w^{4}
$$

As thrust can be expressed as $T=\rho n^{2} D^{4} C_{T}$ and the advance ratio of the propeller is defined as $J=V / n D$, then Eq. (18) can be rewritten as

$$
\begin{gathered}
\left(\frac{2 n^{2} D^{2} C_{T}}{\pi}\right)^{2}=V^{2} w^{2}+2 V w^{3} \cos \alpha_{p}+w^{4} \\
\left(\frac{2 C_{T}}{\pi J^{2}}\right)^{2}=\left(\frac{w}{V}\right)^{2}+2\left(\frac{w}{V}\right)^{3} \cos \alpha_{p}+\left(\frac{w}{V}\right)^{4}
\end{gathered}
$$

According to Eq. (19a), an increase in the propeller frequency $n$, or in revolutions per minute, is associated with an increase in $w$. The same effect can be observed in terms of $J$ from Eq. (19b), where a raise in $J$, or an increase in $V$ relative to $n$ or revolutions per minute, will cause a decrease in $w / V$ and, vice versa, a rise in the ratio of the propeller rotation to wind speed $V$ will incur in an increase in $w / V$. For the particular case of static thrust, where $V=0$, one obtains the classical induced velocity for hover condition $\left.w\right|_{V=0}$,

$$
\left.w\right|_{V=0}=\sqrt{\frac{T}{2 \rho S_{\text {disk }}}}
$$

For $\mathrm{AOA}=0$ deg (i.e., no incidence) and if $T>0$, the only physically valid solution of the four roots for Eq. (18) is $\left.w\right|_{\mathrm{AOA}=0 \text { deg }}$ as (see Ref. [4])

$$
\left.w\right|_{\mathrm{AOA}=0 \text { deg }}=\frac{1}{2} \sqrt{\left(\frac{2 T}{\rho S_{\mathrm{disk}}}\right)+V^{2}}-\frac{V}{2}
$$

Equation (21) can be rewritten as

$$
\left(\frac{w}{V}\right)_{\mathrm{AOA}=0 \mathrm{deg}}=\frac{1}{2} \sqrt{\left(\frac{\left.8 C_{T}\right|_{\mathrm{AOA}=0 \mathrm{deg}}}{\pi J^{2}}\right)+1}-\frac{1}{2}
$$

The derived equations from the classical momentum theory for propellers at a given incidence angle allows one to estimate the theoretical slip-stream angle at the disk $\alpha_{\text {slp }}$ and at the ultimate wake $\alpha_{\text {slpult }}$. As illustrated in Fig. 1b, it can be shown from Eqs. (13), and $(\underline{15 b})$ that

$$
\begin{gathered}
\alpha_{\text {slpult }_{\text {ul }}=} \arcsin \left(\frac{2(w / V) \sin \alpha_{p}}{\sqrt{1+4(w / V) \cos \alpha_{p}+4(w / V)^{2}}}\right) \\
V_{\text {ult }}=V \sqrt{1+4\left(\frac{w}{V}\right) \cos \alpha_{p}+4\left(\frac{w}{V}\right)^{2}}
\end{gathered}
$$

$\alpha_{\text {slp }}$ in Fig. $1 \mathrm{~b}$ at the disk is related to $\alpha_{p}$ and $\varepsilon$ as

$$
\alpha_{\mathrm{slp}}=\alpha_{p}-\varepsilon=\alpha_{p}-\arctan \left(\frac{\sin \alpha_{p}}{\cos \alpha_{p}+(w / V)}\right)
$$

Further analysis shows

$$
\alpha_{\mathrm{slp}}=\arcsin \left(\frac{(w / V) \sin \alpha_{p}}{\sqrt{1+2(w / V) \cos \alpha_{p}+(w / V)^{2}}}\right)
$$

It has been shown that the angle $\varepsilon$ influences $T$ through the entrainment factor $e$. For no incidence flight, $\varepsilon$ equals $\alpha_{p}$ at $0 \mathrm{deg}$. For $\alpha_{p}=90 \mathrm{deg}$, 


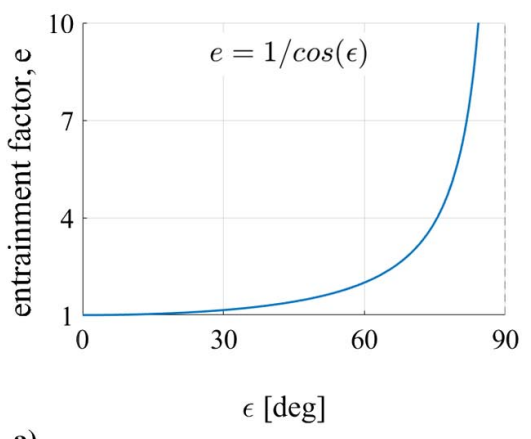

a)

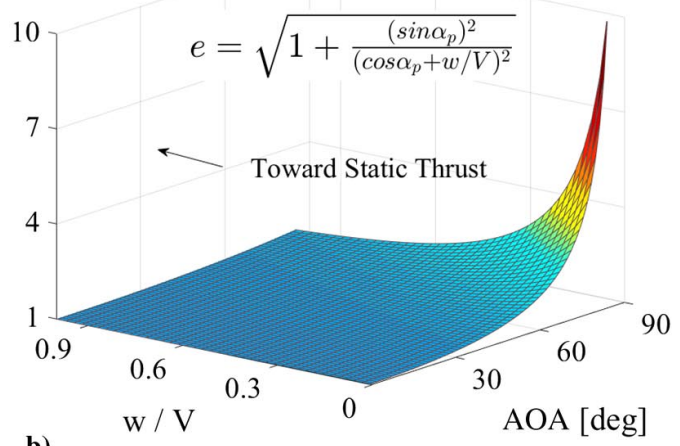

b)

$\mathrm{w} / \mathrm{V}$

AOA $[\operatorname{deg}]$

Fig. 2 Variation of $e$ determined from Eq. (6): a) $e$ as function of $\varepsilon$ and b) $e$ as function of AOA and $w / V$.

$$
\varepsilon=\arctan \left(\frac{V}{w}\right)
$$

Figure 2a illustrates the variation of the entrainment factor $e$ determined by using Eq. (6) with $\varepsilon$. The effects of AOA and $w / V$ on $e$ is shown in Fig. 2b. It can be seen that at low angles of incidence $e$ tends to be unity. The effective area $S_{\text {eff }}$ is similar to the rotor disk area, as the propeller is operating at wind speeds near the axial condition. $S_{\text {eff }}$ is increased with $e$ and therefore with AOA. As AOA approaches $90 \mathrm{deg}$ and $V \cos \alpha_{p}$ tends to zero, $e$ tends to infinity, in theory at very low $w / V$ so as to increase the area $S_{\text {eff }}$ in order to maintain the same finite value of mass flow rate $\dot{m}$ through the rotor, according to Eqs. (1), and (2). In these extreme cases, the angle $\varepsilon$ is approaching $90 \mathrm{deg}$, as $V_{\text {disk }}$ is almost parallel to the rotor disk. The increase in $e$ becomes more relevant at high angles of incidence and at very low $w / V$ values.

\section{B. Entrainment Factor Expansion and Introducing Axial and Wing Equivalent Components of Thrust}

The entrainment factor $e$ can be expanded as a Maclaurin series as

$$
e=\frac{1}{\cos \varepsilon}=1+\frac{\varepsilon^{2}}{2}+\frac{5 \varepsilon^{4}}{24}+\frac{61 \varepsilon^{6}}{720}+\frac{277 \varepsilon^{8}}{8064}+\frac{50521 \varepsilon^{10}}{3628800}+\ldots
$$

By naming the higher-order terms of the rhs of Eq. (28) as $\sum \operatorname{terms}(\varepsilon)$,

$$
e=\frac{1}{\cos \varepsilon}=1+\sum \operatorname{terms}(\varepsilon)
$$

Applying Eq. (16) and using Eq. (29) to replace the entrainment factor leads to the thrust $T$ being determined as

$$
T=2 \rho\left(V \cos \alpha_{p}+w\right) S_{\text {disk }} w+2 \rho\left(V \cos \alpha_{p}+w\right) S_{\text {disk }} w \sum \operatorname{terms}(\varepsilon)
$$

It can be seen that the first term on the rhs of Eq. (30) has the form of an axial thrust, as the disk area is constant and independent of the entrainment factor $e$ for any angle of attack. There is only the component of the incoming wind velocity in the axial direction, with its absolute value changing with $\cos \alpha_{p}$. Thus, that term is named the $T_{\text {axial }}$ component of thrust, and it is defined as

$$
T_{\text {axial }}=2 \rho\left(V \cos \alpha_{p}+w\right) S_{\text {disk }} w
$$

The second term on the rhs of Eq. (30) can be rewritten by expressing $\sum \operatorname{terms}(\varepsilon)=1 / \cos \varepsilon-1$ from Eq. (29) and by using the following relationship that can be obtained from the geometric graph in Fig. 1b:

$$
\left(V \cos \alpha_{p}+w\right)=\frac{V \sin \alpha_{p}}{\sqrt{\left(\frac{1}{\cos \varepsilon}-1\right)\left(\frac{1}{\cos \varepsilon}+1\right)}}
$$

After further simplification, we notice that the resulting expression has the form of the wing lift formula from wing theory $[3,5,7,52]$. Therefore, we define it as the wing equivalent component of thrust $T_{\text {wing }}$ as

$$
T_{\text {wing }}=2 \rho V \sin \alpha_{p} w S_{\text {disk }} \sqrt{\frac{1-\cos \varepsilon}{1+\cos \varepsilon}}
$$

Because it is assumed in the disk actuator theory that the induced speed $w$ is constant across the rotor disk, $T_{\text {wing }}$ can be considered to be equivalent to the lift produced by an elliptic wing subjected to a wind speed of magnitude $V \sin \alpha_{p}$ and having the wing surface area equal to

$$
S_{\text {wing }}=S_{\text {disk }} \sqrt{\frac{1-\cos \varepsilon}{1+\cos \varepsilon}}
$$

The factor multiplying $S_{\text {disk }}$ and defining the equivalent wing area will be regarded as the wing factor $W F$. It can be expressed also as function of $e$ as

$$
W F=\sqrt{\frac{1-\cos \varepsilon}{1+\cos \varepsilon}}=\sqrt{\frac{e-1}{e+1}}
$$

Substituting Eq. (6) on Eq. (35) and simplifying it leads to $W F$ being given as

$$
W F=\frac{\sin \alpha_{p}}{\sqrt{1+2(w / V) \cos \alpha_{p}+(w / V)^{2}}+\cos \alpha_{p}+(w / V)}
$$

Figure 3 a shows the variation of $W F$ with the angle $\varepsilon$. For low $\varepsilon$, $W F$ implies a very small equivalent wing area, vanishing at $\varepsilon=$ $0 \mathrm{deg}$ (where also $\alpha_{p}=0 \mathrm{deg}$ and the wing component vanishes). For $\varepsilon \rightarrow 90 \mathrm{deg}, W F$ tends to unity. It yields then a full wing equivalent area of $S_{\text {disk }}$. It can be seen from Fig. $3 b$ that $W F$ can only reach unity for $w / V \rightarrow 0$, or at high speeds, and at $\mathrm{AOA}=90 \mathrm{deg}$, which corresponds also to $\varepsilon \rightarrow 90 \mathrm{deg}$. For AOA $\neq 0 \mathrm{deg}, W F$ only vanishes asymptotically as $w / V \rightarrow \infty$, when hovering.

In the extreme case, at $\mathrm{AOA} \rightarrow 90 \mathrm{deg}$ and $w / V \rightarrow 0$, where $W F \rightarrow 1$, thrust can be shown as

$$
T=T_{\text {axial }}+T_{\text {wing }} \rightarrow 2 \rho S_{\text {disk }} w^{2}+2 \rho V w S_{\text {disk }}
$$

Here, it can be seen that $T_{\text {axial }}$ takes the form of the static thrust formula and it contributes to total thrust even at forward speeds. $T_{\text {wing }}$ converges to the wing lift formula with full area $S_{\text {disk }}$ in the case of a 

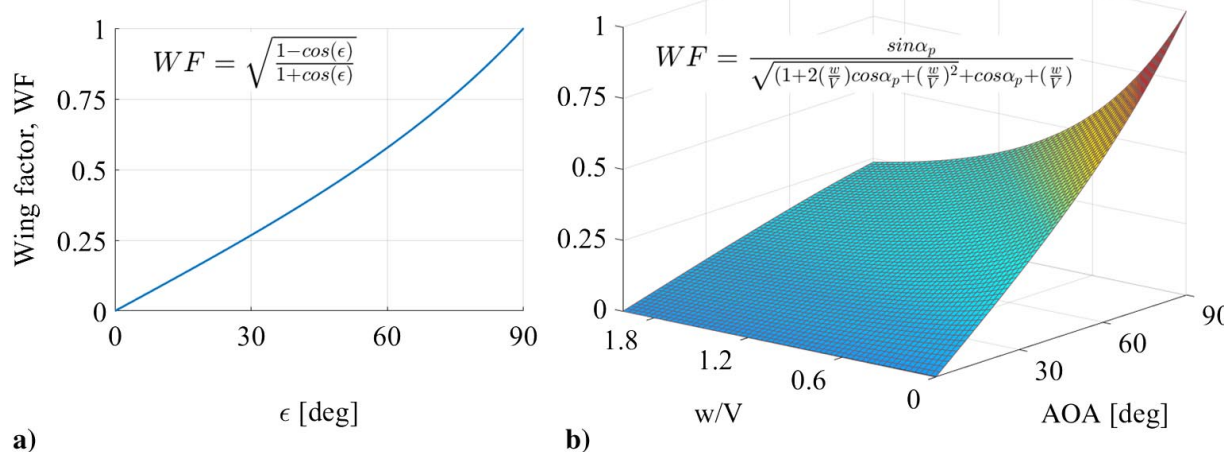

b)

Fig. 3 Variation of $W F$ as determined by Eqs. (35) and (36): a) $W F$ as function of $\varepsilon$ and b) $W F$ as function of AOA and $w / V$.

rotor at fast forward flight $(w \ll V)$, when $T_{\text {axial }}$ is negligible. As $T_{\text {wing }}$ becomes dominant, the propeller behaves as a wing. These two expressions are mentioned by Glauert [7] in the development of his thrust hypothesis in the analysis of helicopters in forward flight. Ariza [22] presents results from 3D numerical simulations showing induced vortices produced by the rotor when at high angles of attack in the same manner as finite wing vortices. It is suggested that a possible explanation for the phenomenon in those conditions is that the propeller could behave as an elliptic wing. Johnson [11] also mentions that the helicopter rotor behaves as a wing at forward speeds and that these two expressions are the limits of thrust when $V \gg w$ and when in hover, where $V=0$. Although he claims that there is no theoretical justification for the approach at intermediate forward speeds, good agreement has been found with measured rotor performance and with vortex theory results, suggesting therefore that it should be accepted for the entire range of speeds. McCormick [5] also mentions the analogy of the propeller at forward flight to an elliptic wing when $w / V \rightarrow 0$ and when $V=0$ to the hover case. The derivations of Eqs. (31) and (33) in this Paper allow us to assume that indeed the two components $T_{\text {axial }}$ and $T_{\text {wing }}$ are always present and comprise the total thrust for the whole operational envelope, at any angle of incidence and velocity.

From Eqs. (31) and (33), it is possible to calculate the ratio $T_{\text {wing }} / T_{\text {axial }}$ to show the contribution of the wing component to the axial component on thrust, according to

$\frac{T_{\text {wing }}}{T_{\text {axial }}}=\frac{2 \rho V \sin \alpha_{p} w S_{\text {disk }} \sqrt{\frac{1-\cos \varepsilon}{1+\cos \varepsilon}}}{2 \rho\left(V \cos \alpha_{p}+w\right) S_{\text {disk }} w}=\tan \varepsilon \sqrt{\frac{1-\cos \varepsilon}{1+\cos \varepsilon}}$

Figure 4 shows the variation of $T_{\text {wing }} / T_{\text {axial }}$ with AOA and $w / V$, determined by Eqs. (6) and (38). The region where $T_{\text {wing }} \geq T_{\text {axial }}$ is highlighted. It can be seen that the contribution of $T_{\text {wing }}$ to the total thrust $T_{\text {wing }}+T_{\text {axial }}$ increases with AOA and with $V$. As $w / V$ is reduced to less than 0.6 and AOA grows higher than $60 \mathrm{deg}$, eventually $T_{\text {wing }}$ overcomes $T_{\text {axial }}$ and becomes dominant, in other words, $T_{\text {wing }} / T_{\text {axial }}>1$.

At high AOA and at high speeds $(w / V \rightarrow 0)$, then $T_{\text {wing }} /$ $T_{\text {axial }} \rightarrow \infty$, so $T$ is composed mainly by $T_{\text {wing. }}$. In those cases, $T \rightarrow$ $2 \rho V w S_{\text {disk }}$. At $V=0$ in hover, it becomes $T=2 \rho S_{\text {disk }} w^{2}$. This agrees well with the findings reported by Glauert [7]. By manipulating Eqs. (35), (36), and (38), it is possible to rewrite $T=T_{\text {axial }}(1+$ $\left.T_{\text {wing }} / T_{\text {axial }}\right)$ as

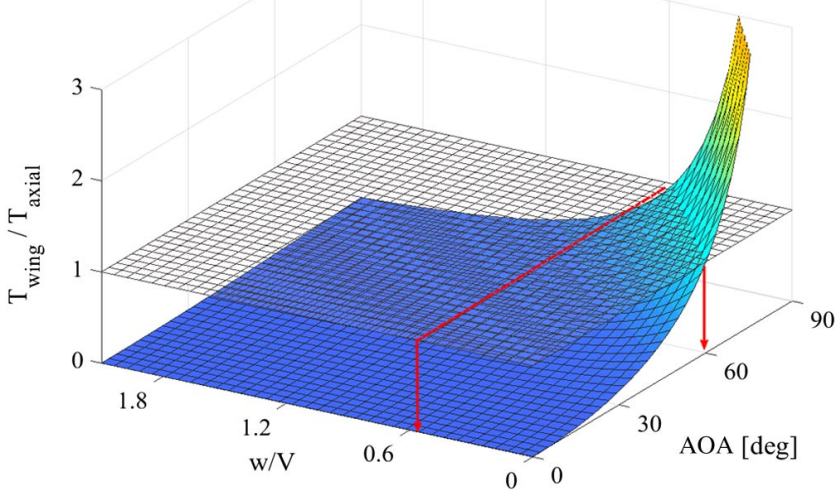

Fig. 4 Variation of $T_{\text {wing }} / T_{\text {axial }}$ with $w / V$ and AOA.

\section{Experimental Wind Tunnel Tests}

\section{A. Experimental Setup and Tests Procedures}

The experimental tests were conducted at the University of Canterbury's closed circuit subsonic wind tunnel that has a rectangular cross section of $0.9 \mathrm{~m}$ in height by $1.20 \mathrm{~m}$ in width, providing maximum test speeds of $60 \mathrm{~m} / \mathrm{s}$. The force balance is a six-axis JR3 45E15A4 sensor capable of measuring the forces and moments in the three-axis $x y z$. It can stand loads of up to $400 \mathrm{~N}$ with nominal accuracy of $\pm 0.25 \%$. A fourth-order Butterworth analog filter with eight different cutoff frequencies ranging from 6.3 to $926 \mathrm{~Hz}$ can be selected via jumper plugs. The default frequency at the wind tunnel is set to $6.3 \mathrm{~Hz}$ for maximum noise attenuation. An aluminum rig with variable AOA settings was manufactured to hold a $6 \mathrm{~mm}$ carbon fiber square rod and the 3D-printed motor/propeller assembly, shown in Fig. $\underline{5}$.

The propeller used is a two-blade HQ that is $6.0 \mathrm{in}$. diameter, $4.5 \mathrm{in}$. pitch, powered by an Ethix TeamBlacksheep Silk V2 2345 Velectric motor. An 80A T-motor electronic speed controller (ESC) is used to control the motor. A power supply continuously provides $15.7 \mathrm{~V}$ and electric current set according to power requirement to maintain a desired revolutions per minute, limited at around 20 A for motor constraints. The input electric current for the motor is measured through a current meter that communicates with data acquisition hardware (DAQs) from National Instruments to a desktop PC prepared with a LabVIEW program that records electric power, current,

$$
T=T_{\text {axial }}\left\{1+\frac{\left(\sin \alpha_{p}\right)^{2}}{\left[\cos \alpha_{p}+\left(\frac{w}{V}\right)\right]\left[\sqrt{1+2\left(\frac{w}{V}\right) \cos \alpha_{p}+\left(\frac{w}{V}\right)^{2}}+\cos \alpha_{p}+\left(\frac{w}{V}\right)\right]}\right\}
$$



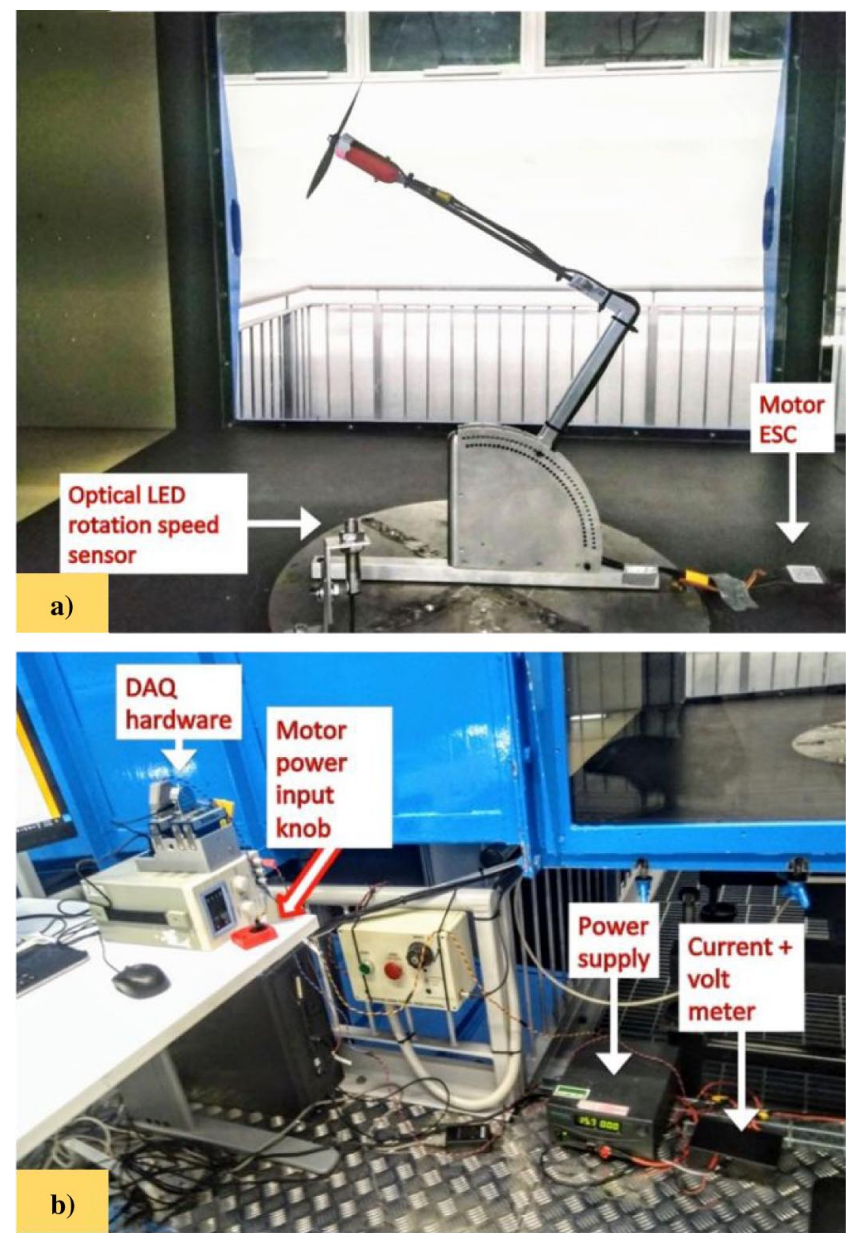

Fig. 5 Wind tunnel settings: a) the propeller test rig and b) experiment hardware.

voltage, and motor revolutions per minute. A Monarch remote optical light-emitting diode (LED) sensor able to measure up to $250,000 \mathrm{rpm}$ from up to $0.9 \mathrm{~m}$ distance, at a maximum of $45 \mathrm{deg}$ angle, is installed at the wind tunnel test section facing the motor that is prepared with a reflective tape on top of half of its circumference. An exponential smoothing filter for revolutions per minute readings was applied in the LabVIEW program. Another LabVIEW program is used for the force measurement and includes a low-pass filter with adjustable cutoff frequencies. Oscillatory nature of thrust, moments, and lateral forces from propellers at incidence are mitigated by the use of the filter. A $1 \mathrm{~Hz}$ cutoff frequency has been chosen throughout the tests in order to achieve more stable average readings. Experimental test data are compiled and analyzed with MATLAB ${ }^{\circledR}$.

Propeller thrust and normal forces reading were acquired at wind tunnel speeds ranging from zero to $25 \mathrm{~m} / \mathrm{s}$ and motor speed rotations from 9,000 to 18,000 rpm. Angles of attack ranged from 0 to $90 \mathrm{deg}$. Electric input motor power is also measured and analyzed for each test condition. For a wind speed of $25 \mathrm{~m} / \mathrm{s}$ and AOA higher than $60 \mathrm{deg}$, it was not possible to reach revolutions per minute higher than 15,000 as power requirement exceeded motor limitations.

\section{B. Experimental Data Postprocessing}

Before the experimental tests, the holding set without the propeller was exposed to the same wind speeds and AOA values as of the propeller tests in order to evaluate the resistance of the rig. The forces readings acquired were fitted to spline surface functions. These are netted from the final propeller tests readings. The extra effect of the propeller slip stream on the holding sting was not evaluated, and the total net thrust could be expected to be somewhat higher. However, the slip stream is expected to impinge only on the sting behind the propeller and not on the entire holding set.

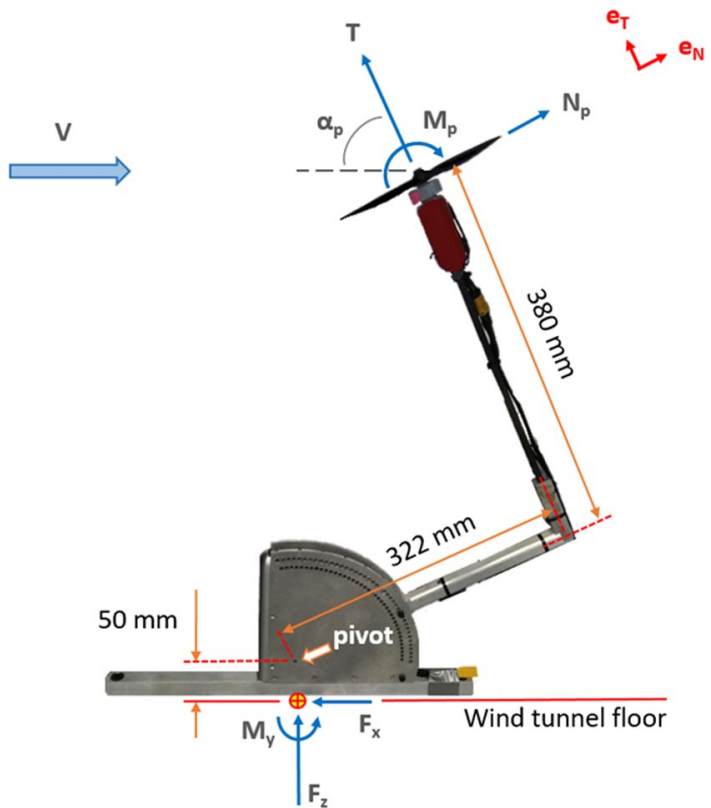

Fig. 6 Scheme of forces for the propeller wind tunnel tests.

Equations (40a) and (40b) enable the calculation of $T$ and $N_{p}$ from forces $F_{x}$ and $\overline{F_{z}}$ measured at the wind tunnel tests, according to the scheme depicted in Fig. 6. The analysis of experimental test data is performed by using the thrust values acquired for every test condition and subsequently inputting those into Eq. (18) to calculate the associated induced speeds $w$. Once $w$ is obtained, then all the remaining calculations can be performed,

$$
\begin{aligned}
& F_{x}=\left(\cos \alpha_{p}\right) T-\left(\sin \alpha_{p}\right) N_{p} \\
& F_{z}=\left(\sin \alpha_{p}\right) T+\left(\cos \alpha_{p}\right) N_{p}
\end{aligned}
$$

The experimental data were acquired for around $20 \mathrm{~s}$ per experimental test. Batches of three tests were performed for every tested condition, in other words, $R P M, A O A$, and $V$, comprising around 80 points per condition. The experimental measurements were averaged, and the precision error evaluated as one standard deviation and presented in percentage terms relative to the average for $V$ and $R P M$ and in absolute terms for the forces $F_{x}$ and $F_{z}$. Presenting the forces errors relative to very small average force values, in many experimentally tested cases, would incur in meaningless very high percentage values. A total of 182 tested conditions were measured. The figure shown in Appendix $\mathrm{C}$, presents the measurements errors obtained for all tested conditions. The maximum error for $V$ was around $5 \%$, although the vast majority of the tests showed errors below $2.0 \%$. The maximum error for RPM was $0.8 \%$, for $F_{x}, 0.14[\mathrm{~N}]$, and for $F_{z}, 0.65[\mathrm{~N}]$. The errors for $T$ were inferred from $F_{x}$ and $F_{z}$ error propagation, according to $\sigma_{T}=$ $\sqrt{\left(\cos \alpha_{p} \sigma_{F x}\right)^{2}+\left(\sin \alpha_{p} \sigma_{F z}\right)^{2}}$. The cumulative distribution function for all $\sigma_{T}$ points is presented in the subgraph $f$ in Appendix $\mathrm{C}$, where it is shown that for $85 \%$ of the cases $\sigma_{T}<0.1 \mathrm{~N}$ and that the measurement errors tend to a log-normal distribution behavior as verified by a corresponding data fit.

\section{Results and Discussions}

\section{A. Thrust Measurements Analysis}

Figure 7a shows thrust performance of the propeller as a function of $R P M$ at different wind speeds, at no incidence (AOA $=0 \mathrm{deg}$ ). As expected from the momentum theory, the freestream velocity increase at constant $R P M$ (or a raise in $J$ ) will cause a reduction in the available thrust [5]. It is also noticeable from propeller tests results presented in previous works [19-23,53,54], and the thrust exhibits a parabolic variation with $R P M$ as in Refs. [19-23,53-56]. Figure 7b illustrates the influence of AOA on thrust for a constant wind speed 


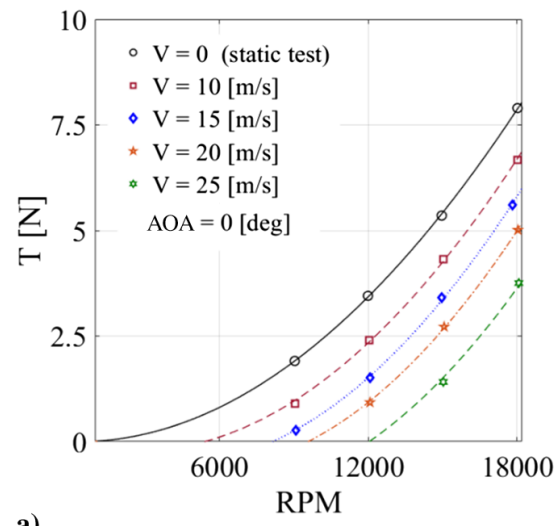

a)

Fig. 7 Variation of thrust $T$ with $R P M:$ a) as $V$ is set to five different values and AOA $=0$ deg and b) as $V=20 \mathrm{~m} / \mathrm{s}$ and $\mathrm{AOA}$ is set to four different values.

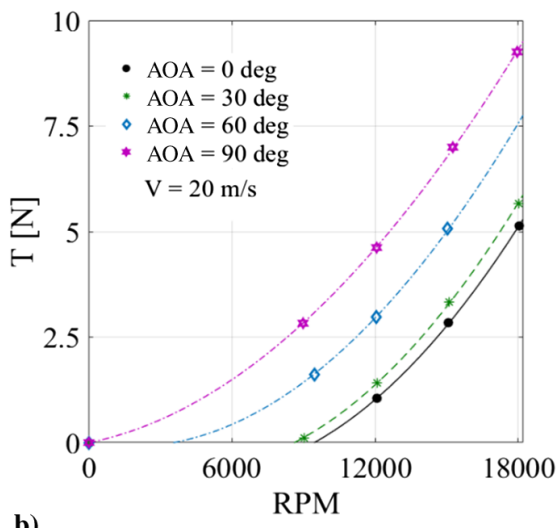

)

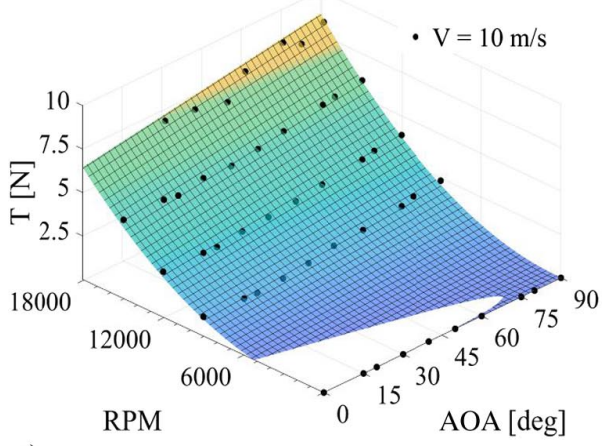

a)

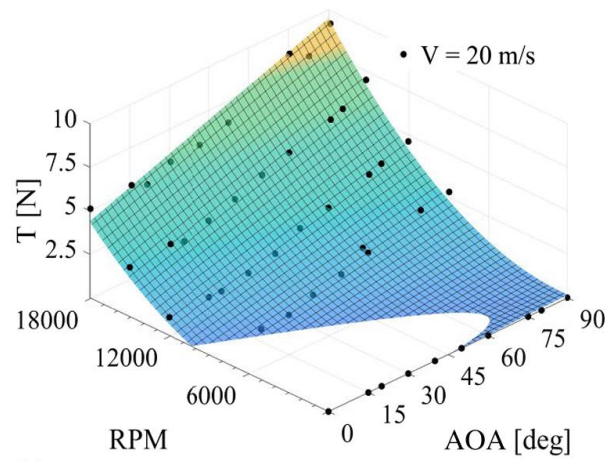

b)

Fig. 8 Thrust $T$ varied with AOA and RPM: a) $V=10 \mathrm{~m} / \mathrm{s}$ and b) $V=20 \mathrm{~m} / \mathrm{s}$.

$V=20 \mathrm{~m} / \mathrm{s}$. The thrust $T$ is increased, as the angle of incidence rises. This finding is consistent with the previous concluding remarks in Refs. [19-23].

Figure 8 presents the thrust measured from the propeller as function of AŌA and $R P M$, for a) $V=10 \mathrm{~m} / \mathrm{s}$ and b) $V=20 \mathrm{~m} / \mathrm{s}$. Again, from both graphs at $\mathrm{AOA}=0 \mathrm{deg}$, it can be seen that the thrust is reduced with the increase of $V$ at $18,000 \mathrm{rpm}$ and $V=$ $10 \mathrm{~m} / \mathrm{s}$ in Fig. 8a, in which $T=6 \mathrm{~N}$, while in Fig. 8b for $V=$ $20 \mathrm{~m} / \mathrm{s}, T=4 \overline{\mathrm{N}}$ approximately. As AOA rises, $T$ increases in both cases. However, the slope of thrust increase with AOA $\partial T / \partial \alpha_{p}$ is higher in the case of the higher speed. Another interesting observation from Fig. 8 is that at low AOA and for airspeeds higher than zero no thrust is available, which is also seen in Fig. 7. In that region, the propeller will perform in a windmill state until a sufficient value of $R P M$ is achieved. For example, in Fig. 8a in which $V=10 \mathrm{~m} / \mathrm{s}$, no thrust is generated before the propeller reaches around $5000 \mathrm{rpm}$ at low angles of attack. For the case of $V=20 \mathrm{~m} / \mathrm{s}$ in Fig. $8 \mathrm{~b}$, the value 7 required is $9500 \mathrm{rpm}$ at no incidence, and the region of no thrust achievable $(T<=0)$ is wider, reaching up to AOA $\approx 60 \mathrm{deg}$. However, as AOA is increased, less $R P M$ is required to achieve some thrust in both cases.

Figure $\underline{9}$ shows how thrust $T$ is varied with AOA, for $V=10, V^{-}=15$, and $V=20 \mathrm{~m} / \mathrm{s}$ and rotation speeds of 12,000 , 15,000 , and $18,000 \mathrm{rpm}$. In all tested cases, the thrust $T$ is found to always increase with AOA, more noticeably for AOA $>20$ deg. A higher slope $\partial T / \partial \alpha_{p}$ is associated with a higher $V$, for a given $R P M$. Therefore, the analysis here means to be extended also to the advance ratio $J$, as an increase in velocity, at a given constant $R P M$, represents also an increase in $J$, and vice versa for a decrease. This difference in slopes will cause a change in thrust sensitivity to $V$ at high angles of incidence; while at low angles $T$ decreases with $V(\partial T / \partial V<0)$, for AOA around $60 \mathrm{deg}$ and over, $T$ eventually starts to increase with increased $V(\partial T / \partial V>0)$. Similar results for $C_{T}$ growing with AOA

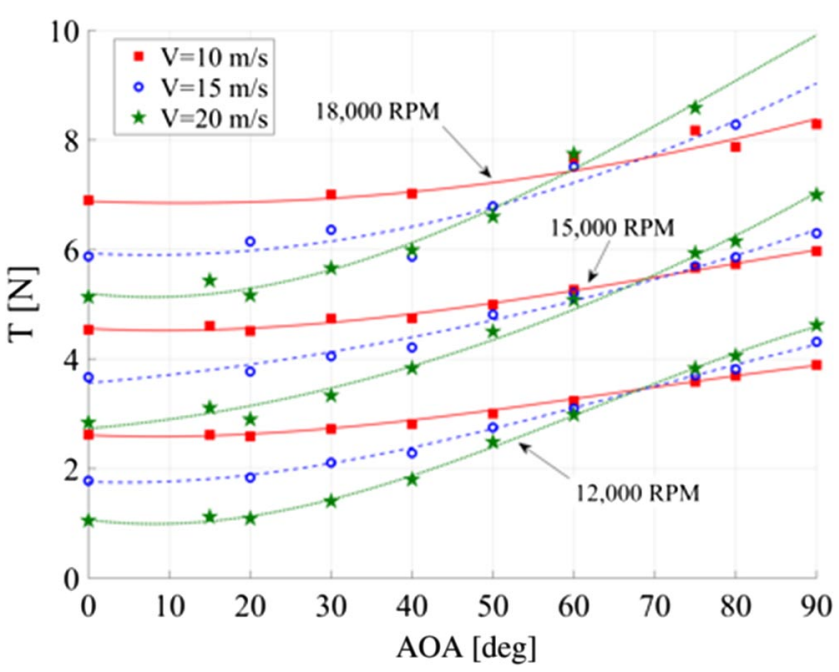

Fig. 9 Variation of $T$ with AOA at different RPM, as $V$ is set to three different values

and the inversion of behavior at a higher AOA are found in the experimental tests in Refs. [21-23].

Figure $10 \mathrm{a}$ shows the thrust $T$ contour surface at $15,000 \mathrm{rpm}$. The analysis here, at constant $R P M$, again means that an increase or decrease in $V$ is also associated with a corresponding increase or decrease in $J$. Again, at a low AOA value, $T$ is decreased as $V$ is growing. However, at a high AOA value, beyond $60 \mathrm{deg}$, the thrust variation behavior inverts to increase with $V$. Also, $T$ is always increasing with AOA at constant speeds. $T_{\text {axial }}$ is presented in Fig. 10b, and $T_{\text {wing }}$ is depicted in Fig. 10c, calculated according to 

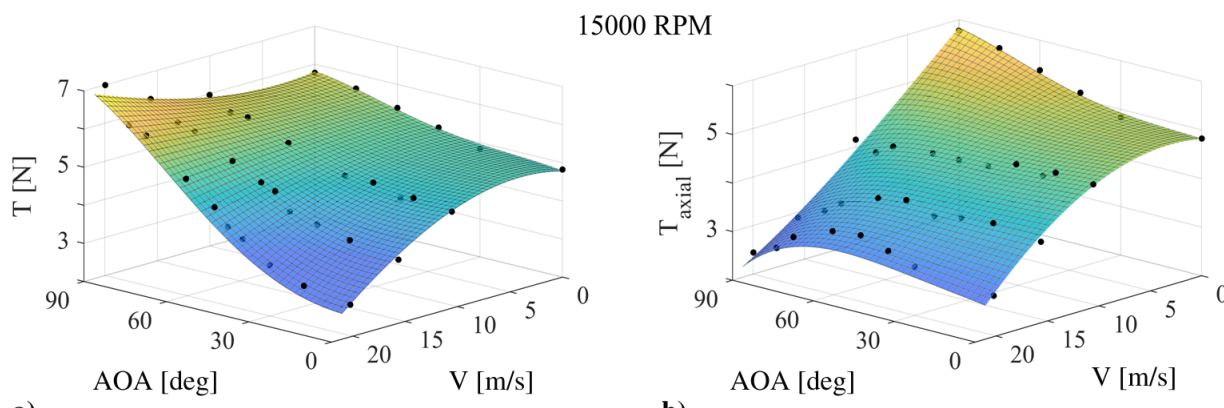

a)

b)

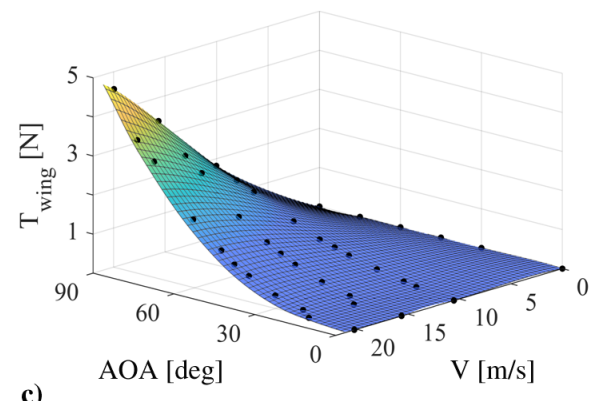

c)

Fig. 10 Variation of the thrust $T, T_{\text {axial }}$, and $T_{\text {wing }}$ with $A O A$ and $V:$ a) $T$ measured at $15,000 \mathrm{rpm}$, b) calculated $T_{\text {axial }}$, and c) calculated $T_{\text {wing }}$.

Eqs. (17), (31), and (33). $T_{\text {axial }}$ behaves in the same way for all angles of incidence (AOA), decreasing as $V$ is increased. $T_{\text {wing }}$ is rising with $V$ and AOA, peaking at $\mathrm{AOA}=90 \mathrm{deg}$ and at higher speeds. The slope of $T_{\text {wing }}$ increase with $A O A$, $\left(\partial T_{\text {wing }} / \partial \alpha_{p}\right)$ also grows with $V$. Note in Figs. $10 \mathrm{a}$ and $\underline{10 \mathrm{~b}}$ the slight increase in $T$ with AOA for $V=0$ (static thrust tests performed at different AOA) is probably associated with ground effects from the wind tunnel. The expected behavior would be the same static thrust at all incidence angles.

Figure 11 shows the measured $T$ and its components $T_{\text {axial }}$ and $T_{\text {wing }}$ calculated for the cases of fixed velocities $V=10 \mathrm{~m} / \mathrm{s}$,

- $\mathrm{T}$ measured $\diamond \mathrm{T}$ axial component $\square \mathrm{T}$ wing component

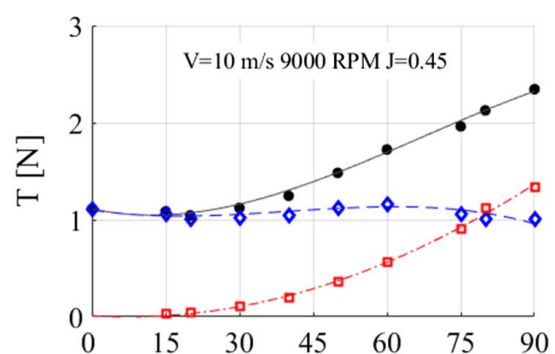

a)

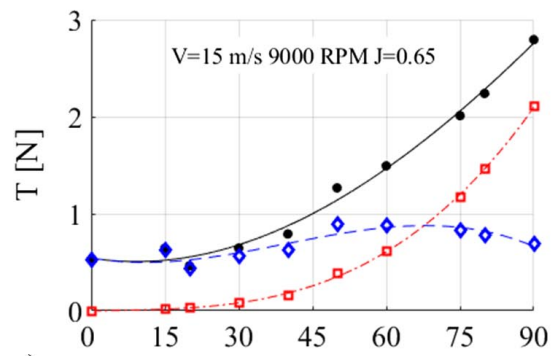

c)

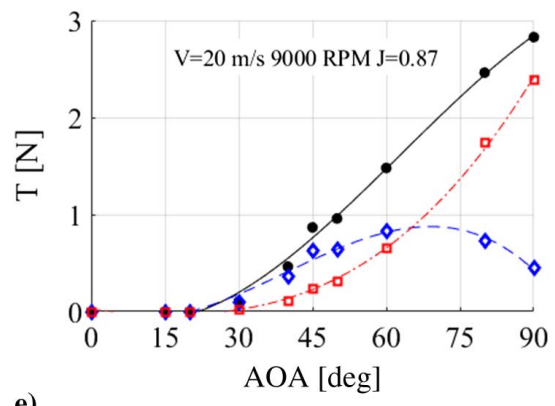

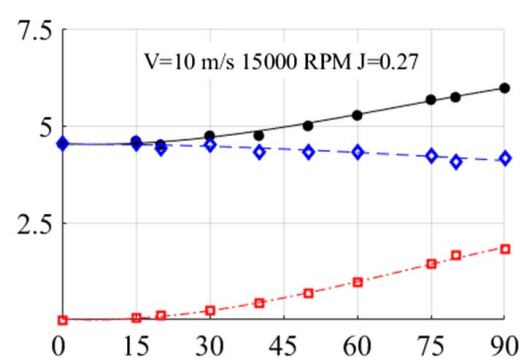

b)

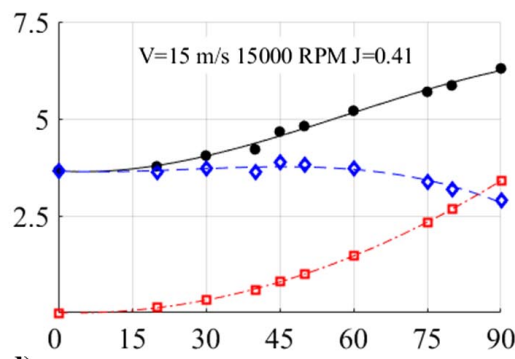

d)

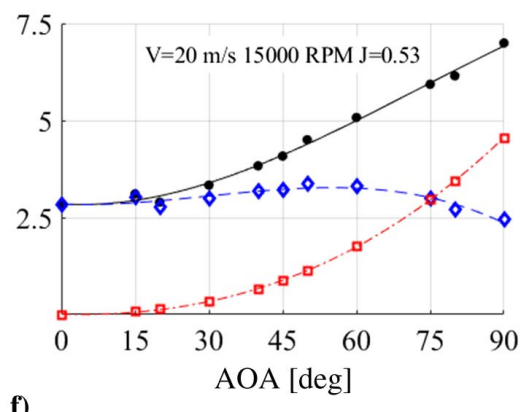

f)

Fig. $11 T$ measured, $T_{\text {axial }}$, and $T_{\text {wing }}$ calculated according to Eqs. (31) and (33). 
$V=15 \mathrm{~m} / \mathrm{s}, V=20 \mathrm{~m} / \mathrm{s}$ and RPM values of 9000 and 15,000. The increase of thrust with AOA $\left(\partial T / \partial \alpha_{p}>0\right)$ follows to a great extent the behavior of $T_{\text {wing }}\left(\partial T_{\text {wing }} / \partial \alpha_{p}>0\right)$ from which it can be inferred the wing component to be mostly the reason for the phenomenon. Also, it can be observed from Figs. 11b, 11d, 11f and 11a, 11c, 11e that as $V($ and $J)$ is increased the sensitivity slopes of $\overline{T_{\text {wing }}} \overline{\text { to } \mathrm{AOA}}$ are also increased. $T_{\text {wing }}$ contribution below $\mathrm{AOA} \approx 30 \mathrm{deg}$ is negligible even at high advance ratios $J$ as the wing factor $W F$ and $\sin \alpha_{p}$ are low at small angles. At $\mathrm{AOA}=0 \mathrm{deg}, T_{\text {wing }}$ is vanished, and $T=T_{\text {axial }}$, which formula becomes Eq. (16) with $e=1$, where the 18 model reverts to the classic momentum theory with no incidence. $T_{\text {wing }}$ relevance starts at AOA $>30 \mathrm{deg}$, and a high $J$ value causes the contribution of $T_{\text {wing }}$ relative to $T_{\text {axial }}$ to become very important in the region around $\mathrm{AOA} \approx 60 \mathrm{deg}$, where $T_{\text {wing }}$ eventually surpasses $T_{\text {axial }}$ as illustrated in Figs. 11a-11f. This can only be observed for $w / V$ lower than around $0.6 \overline{(\text { see }} \overline{\text { Fig. }}$. 4), which is associated with a higher $J$ [see Eq. (19b)]. At very high angles and as $J$ is increased, $T_{\text {wing }}$ composes an ever-larger part of $T$. Note also that as $J$ is increased (decreased $w / V$ ) the crossing of the two components is possible at a lower angle than $90 \mathrm{deg}$, toward $60 \mathrm{deg}$ (Figs. 11a, 11c, $\underline{11 \mathrm{e}}$ and $11 \mathrm{~b}, 11 \mathrm{~d}, 11 \mathrm{f})$. This is in accordance with the theoretical prediction behaviors as shown in Fig. 4.

As $T_{\text {axial }}$ is predominant at angles $\overline{\mathrm{AOA}}<60 \mathrm{deg}, T$ is decreased with increasing speed (and $J$ ), and accordingly $(\partial T / \partial V<0)$. For higher angles, $T$ is growing with $V(\partial T / \partial V>0)$ (see Fig. 11a, 11c, $11 \mathrm{e}$ at $9000 \mathrm{rpm}$ and Figs. 11b, 11d, $11 \mathrm{f}$ at 15,000 rpm). The inversion from negative to positive sensitivity (see the figure in Appendix B) could be explained as the influence of $T_{\text {wing }}$ on $T\left(\partial T_{\text {wing }} / \partial V>\overline{0}\right.$. $)$ being predominant over $T_{\text {axial }}\left(\partial T_{\text {axial }} / \partial V<0\right)$ in that region. In Ref. [21], several experimental tests were performed for $C_{T}$ vs $J$ and AOA, ranging from 0 to 85 deg on three different full-scale propellers at many different blade pitch angles. They found the inversions of slope $\left(\partial C_{T} / \partial J\right)$ occurring at angles of incidence ranging from around 50 to $75 \mathrm{deg}$, depending on the blade angle configuration. It is interesting to note that this inversion is in the region of high AOA, where the theory predicts that $T_{\text {wing }}$ and its effects begin to overcome $T_{\text {axial }}$ if under a large enough velocity $V$ (high $J$ ) (see Fig. 4). A possible explanation for this would be that different blade configurations would have different slope sensitivities of $T$ to $V(\partial T / \partial V)$ as different propellers should have different $T$ surfaces vs $w / V$ and AOA, and so $\left(\partial T_{\text {wing }} / \partial V>\right.$ $\partial T_{\text {axial }} / \partial V$ ) would happen at different angles for different propellers. However, we assume that for any propeller the relation between $T_{\text {wing }}$ and $T_{\text {axial }}$ must follow Eq. (38), as illustrated in Fig. 4, to fulfil the momentum theory assumptions.

$T_{\text {axial }}$ is relatively insensitive to AOA at low advance ratios (up to $J=0.53$ for the propeller tested) and up to angles around $60 \mathrm{deg}$ (see Figs. $11 \mathrm{a}, 11 \mathrm{~b}, 11 \mathrm{~d}$, and $11 \mathrm{f})$. A decrease in $T_{\text {axial }}$ at higher AOA is noticed in all cases, being more accentuated as $J$ is growing $(w / V$ diminishing), where also $T_{\text {wing }}$ growth is more intense. At higher advance ratios, $T_{\text {axial }}$ is increased with AOA to peak at around $60 \mathrm{deg}$ for the propeller studied (see Figs. $11 \mathrm{c}$ and $11 \mathrm{f}$ ). As $T_{\text {wing }}$ tends to be the main component of $T$ at high $\bar{J}$ and $\mathrm{A} \overline{\mathrm{OA}}$, the thrust formula is indeed reduced to the second term of Eq. (37) or $T \rightarrow 2 \rho V w S_{\text {disk }}$ as predicted by Glauert [7] and described in Ref. [11] for helicopterrotors at high translational speeds (high $J$ ), when the propeller behaves as a wing.

\section{B. Simplified Formula for Estimating $T$ at Incidence from Data at $\mathrm{AOA}=0 \mathrm{deg}$}

The thrust measurements obtained previously from the wind tunnel experimental tests at any AOA allow us to calculate $w / V$ from Eq. (18) and estimate $T_{\text {axial }}$ and $T_{\text {wing }}$ at any point. However, it is not possible to measure these two components separately except in the extreme cases of hover and at high $V$ (high $J$, low $w / V$ ), at AOA $\rightarrow 90 \mathrm{deg}$, where $T \rightarrow T_{\text {wing }}$ formula according to Eq. (37) in accordance with Glauert [7]. To prove the validity of the theory, we assume the values of thrust measured at $\mathrm{AOA}=0 \mathrm{deg}$ and calculate the value of $w / V$ from either Eqs. (18) or (19), extrapolating it to any AOA, but using $T$ obtained at $\overline{\mathrm{AOA}}=\overline{0} \mathrm{deg}$. This assumption

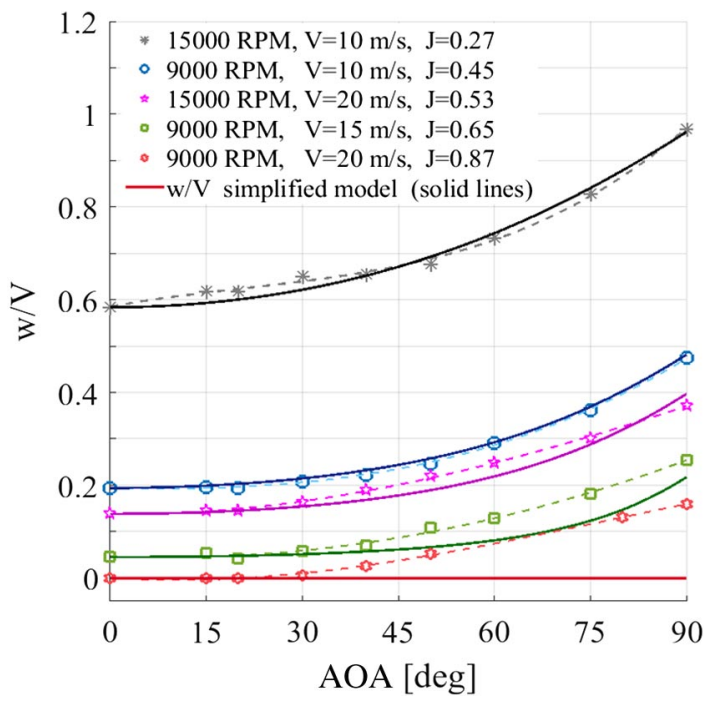

Fig. 12 The $w / V$ obtained from $T$ data at $A O A$ compared to $w / V$ estimated from $\left.T\right|_{\mathrm{AOA}=0 \mathrm{deg}}$ and extrapolated to other AOA values (solid lines).

comes from the fact that $T_{\text {axial }}$ is relatively insensitive to $A O A$ as seen in Fig. 11 unless at high speeds. Figure 12 illustrates $w / V$ calculated from practically measured $T$ data for all angles against the approximation model of $w / V$ in solid lines. It is seen that there is a good match of the simplified model with the measurement data down to $w / V=0.2$ or $J$ up to 0.53 . For $J>0.53$, there is a detachment past $\mathrm{AOA}=30 \mathrm{deg}$ that grows with $J$. At $J=0.87$ and AOA $=0 \mathrm{deg}$, the propeller is windmilling and $\left.T\right|_{\mathrm{AOA}=0 \mathrm{deg}}=0$ as in Fig. 11e, so the model is not suitable as it predicts $w=0$ for all angles.

Figure 13 presents the results obtained from actual thrust measurements against predicted $T$ through the simplified model according to Eq. (39), using the values of $T$ at $\mathrm{AOA}=0 \mathrm{deg}$. Also, $w / V$ projected to be used in Eq. (39) is obtained using $T$ at AOA $=0 \mathrm{deg}$ in either Eqs. (18) or (19) as illustrated in Fig. 12. As the simplified model relies on a projection of thrust at $\mathrm{AOA}=\overline{0} \mathrm{deg}$ and as $T_{\text {axial }}$ is relatively constant up to AOA around $60 \mathrm{deg}$, at low advance ratios (see Fig. 11 for $J<0.53$ ), good agreement is observed between the model and the actual thrust (see Figs. 13a, 13b, and 13d). In those tested cases, $w / V$ projected from the simplified model is very close to $w / V$ obtained from experimental data (see Fig. 12). At around AOA $>70 \mathrm{deg}$, the model overestimates the practical test data. This should be expected as the model assumes a $T_{\text {axial }}$ constant for all incidence angles, but a decrease in that component at a higher AOA can be observed from Figs. 11a, 11b, and 11d.

A small detachment of $\overline{w / V}$ projected by the model, from measured $w / V$ is seen at $J=0.53$ in Fig. 12, which will cause the start of the detachment of $T$ estimated by the model from the real $T$ measured, as in Fig. 13f. As $J$ grows, the detachment of $T$ projected by the model increases as in Fig. 13c until the model eventually loses validity as in Fig. 13e. This happens when $T_{\mid \mathrm{AOA}=0 \text { deg }}$ measured at no incidence is vanished (windmill/brake state), alongside $w / V$, which is used by the model to project $T$ at incidence. The growing detachments of the model $w / V$, from $w / V$ calculated based on the real experimental data, occur in consonance with $T_{\text {axial }}$ no longer being relatively constant and similar to $T_{\mid \mathrm{AOA}=0 \mathrm{deg}}$ at angles up to around $60 \mathrm{deg}$, for increasing $J$ values. In these cases, the $T_{\text {axial }}$ growth with AOA is not captured by the simplified model that underestimates $T$ up to those angles.

\section{Slip-Stream Parameters}

Table 1 summarizes the calculation of the relevant angles and velocities, as depicted in Fig. 1 , from the experimental data. At given $A O A, R P M$, and $V$ conditions and from the thrust $T$ measured, calculations are performed according to Eqs. (4b), (6), (15b), (18), (23), (24), (26), (1), and (33). $T_{\text {wing }}$ and $T_{\text {axial }}$ are also presented at different flow conditions. 
- $\mathrm{T}-\mathrm{T}$ simplified model

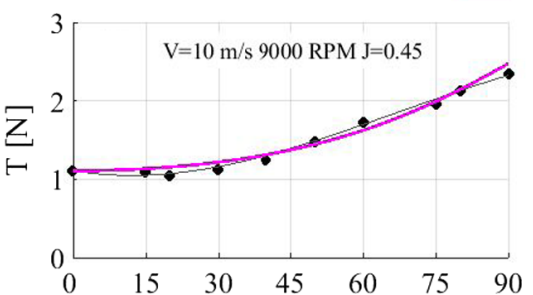

a)

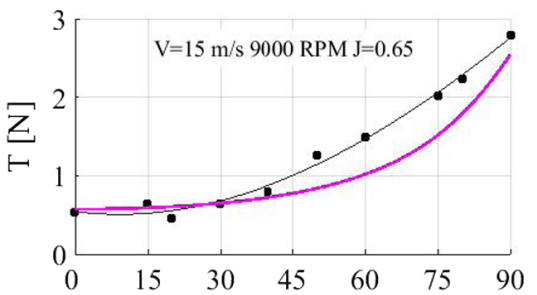

c)

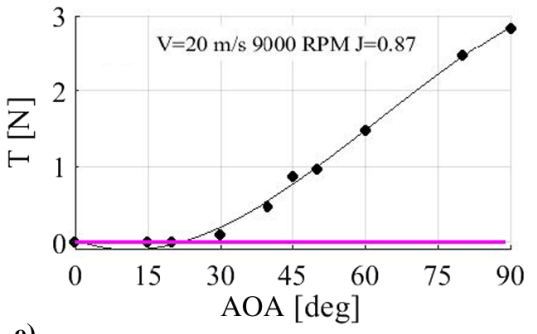

e)

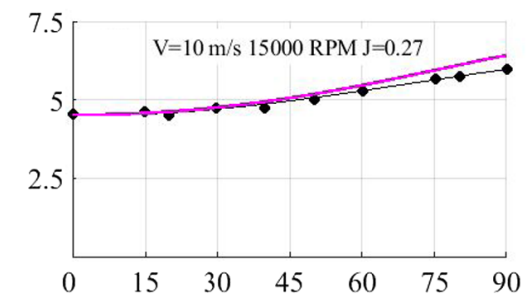

b)

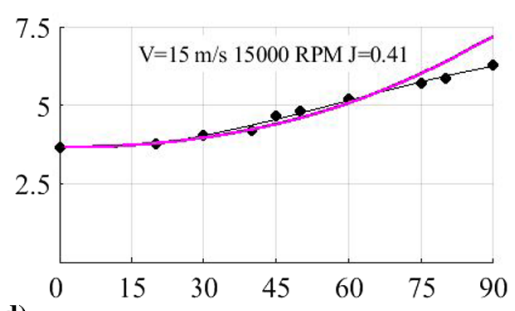

d)

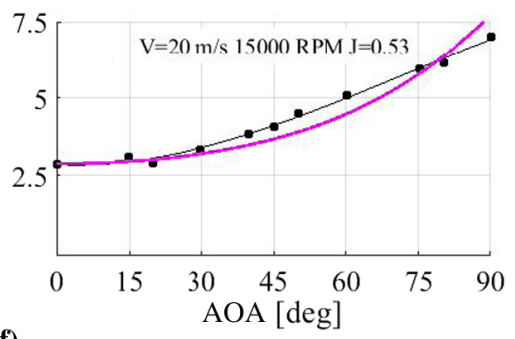

f)

Fig. $13 T$ measured vs simplified model projected from $T_{\mid \mathrm{AOA}=0 \mathrm{deg}}$ on Eqs. (18) and ( $\underline{\text { 39) }}$.

Table $1 T$ measured, $T_{\text {wing }}$, and $T_{\text {axial }}$ calculated from the theory and slip-stream parameters

\begin{tabular}{lrcccccccccccc}
\hline \hline AOA, deg & $R P M$ & $V, \mathrm{~m} / \mathrm{s}$ & $J$ & $w / V$ & $T, \mathrm{~N}$ & $T_{\text {axial }, N}$, & $T_{\text {wing }}, \mathrm{N}$ & $e$ & $\varepsilon, \operatorname{deg}$ & $\alpha_{\text {slp }}, \operatorname{deg}$ & $\alpha_{\text {slp_ult }}, \operatorname{deg}$ & $V_{\text {disk }}, \mathrm{m} / \mathrm{s}$ & $V_{\text {ult }}, \mathrm{m} / \mathrm{s}$ \\
\hline 30 & 17864 & 10.4 & 0.23 & 0.832 & 7.001 & 6.715 & 0.285 & 1.0 & 16.4 & 13.6 & 18.8 & 18.4 & 26.8 \\
30 & 14989 & 15.5 & 0.41 & 0.301 & 4.050 & 3.723 & 0.327 & 1.1 & 23.2 & 6.8 & 11.2 & 19.7 & 24.0 \\
30 & 9024 & 20.1 & 0.87 & 0.006 & 0.103 & 0.089 & 0.014 & 1.2 & 29.8 & 0.2 & 0.3 & 20.2 & 20.3 \\
60 & 17802 & 10.5 & 0.23 & 0.938 & 7.668 & 6.569 & 1.100 & 1.2 & 31.1 & 28.9 & 40.0 & 17.6 & 26.6 \\
60 & 15002 & 15.7 & 0.41 & 0.386 & 5.204 & 3.721 & 1.483 & 1.4 & 44.4 & 15.6 & 25.7 & 19.5 & 24.2 \\
60 & 9074 & 19.9 & 0.86 & 0.081 & 1.480 & 0.824 & 0.655 & 1.8 & 56.1 & 3.9 & 7.4 & 20.8 & 21.7 \\
90 & 17865 & 10.3 & 0.23 & 1.155 & 8.289 & 6.267 & 2.022 & 1.3 & 40.9 & 49.1 & 66.6 & 15.8 & 26.0 \\
90 & 14861 & 15.6 & 0.41 & 0.518 & 6.296 & 2.896 & 3.400 & 2.2 & 62.6 & 27.4 & 46.0 & 17.6 & 22.5 \\
90 & 8979 & 19.9 & 0.87 & 0.160 & 2.828 & 0.446 & 2.382 & 6.3 & 80.9 & 9.1 & 17.7 & 20.2 & 20.9 \\
\hline \hline
\end{tabular}

For $\mathrm{AOA}=30 \mathrm{deg}$, the theoretical entrainment factor $e$ is close to unity, as expected from the momentum theory. For increasing $V$ values, $\alpha_{\text {slp }}$ gets smaller as it is harder for the propeller to turn the flow, especially at low $R P M$ (high $J$, low $w / V$ ), which is in agreement with Ref. [22], and if AOA is high, so is $e$ whenever $\alpha_{\text {slp }}$ is small. The angle $\alpha_{\text {slp-ult }}$ is always greater than $\alpha_{\text {slp }}$ at the disk as $w_{\text {ult }}$ is higher than $w$ [see Fig. 1 and Eq. (15b), $w_{\text {ult }}=2 w$ ]. At high AOA, the contribution of $T_{\text {wing }}$ becomes more relevant, and for $w / V$ values lower than 0.6 and angles higher than $60 \mathrm{deg}, T_{\text {wing }}$ eventually surpasses $T_{\text {axial }}$ as in the last two cases where $\mathrm{AOA}=90 \mathrm{deg}$ and $w / V=0.518$ and 0.16 . Note the entrainment factor $e$ is also high $(e=2.2$ and $e=6.3)$ accompanied by a high angle $\varepsilon$ in these instances.

Figure 14a depicts the behavior of $\alpha_{\text {slp }}$ as a function of $J$ and AOA for the propeller being tested in this Paper. It is seen that for static tests

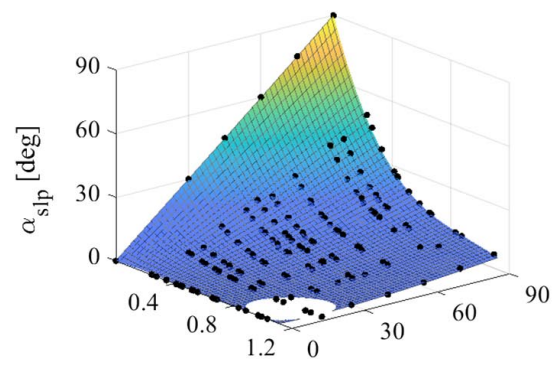

a)
Advance ratio, $\mathrm{J}$

AOA [deg]

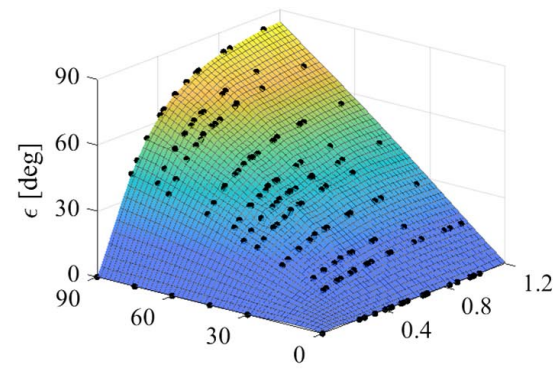

b)

Fig. 14 Angles variation with $J$ and AOA: a) slip-stream angle at the disk $\alpha_{\text {slp }}$ and b) angle $\varepsilon$, between $V_{\text {disk }}$ and thrust $T$. 
$(J=0) \alpha_{\text {slp }}=$ AOA. At no incidence (AOA $\left.=0 \mathrm{deg}\right), \alpha_{\text {slp }}$ also vanishes as the flow is axial. As $J$ is increased, $\alpha_{\text {slp }}$ tends to diminish, and for a constant $J, \alpha_{\text {slp }}$ grows with increased AOA. Figure $\underline{14 \mathrm{~b}}$ shows that the angle $\varepsilon$ is varied with AOA and with $J$. For static test $(J=0), \varepsilon=0 \mathrm{deg}$ as $\boldsymbol{w}$ is aligned with $\boldsymbol{T}$. At AOA $=90 \mathrm{deg}, \varepsilon$ tends asymptotically to 90 deg with the growth of $J$ (which implies growth in $V / w$ ). The analysis of $\varepsilon$ is of interest as it defines the entrainment factor $e$ and the wing factor $W F$ and therefore is directly related to $T_{\text {wing }}$, as indicated in Eqs. (33) and (35) and shown in Fig. $\underline{3}$. The maximum $\varepsilon$ is associated with the highest $T_{\text {wing }}$, which occurs at high $J$ (low $w / V$ values) and high angles of incidence (see Fig. 10c).

\section{Conclusions}

In this Paper, a series of experimental tests was conducted to investigate the aerodynamic performance of a two-blade propeller operating at angles of incidence (AOA) ranging from 0 to $90 \mathrm{deg}$. This is done in in a closed-loop wind tunnel at University of Canterbury. An alternative approach on analyzing aerodynamic thrust performance is proposed. It is based on the classical momentum theory by defining a theoretical entrainment factor $e$ accounting for the mass flow rate through an enhanced area of the rotor disk to equalize Glauert's hypothesis mass flow definition. The factor $e$ is found to depend on the angle $\varepsilon$, defined as the angle between $\boldsymbol{V}_{\text {disk }}$ and the thrust vector $\boldsymbol{T}$. It is shown mathematically that the thrust $T$ consists of two components: one is the axial component $T_{\text {axial }}$, and the other is the wing lift equivalent component $T_{\text {wing }}$.

$T_{\text {axial }}$ behaves similarly to a propeller operating at no incidence under an axial stream of magnitude $V \cos \alpha_{p}$. Therefore, for a given $R P M$, it is decreased with increasing airspeed $\left(\partial T_{\text {axial }} / \partial V<0\right)$, for all angles of incidence. $T_{\text {axial }}$ is shown to be not so sensitive to AOA at low airspeeds (low $J$ ), especially at $\mathrm{AOA}<60 \mathrm{deg}$, while at high airspeeds (high $J$ ), it grows with the angle of incidence up to $\mathrm{AOA} \approx 60 \mathrm{deg}$. At higher angles, $T_{\text {axial }}$ is decreased with increasing AOA. This decrease is more intense at higher speeds.

$T_{\text {wing }}$ provides the equivalent lift of an elliptic wing under velocity $V \sin \alpha_{p}$ of magnitude with a variable area equal to $S_{\text {disk }}$ multiplied by a factor $W F$. This factor ranges from zero at $\mathrm{AOA}=0 \mathrm{deg}$ to $W F \rightarrow 1$ as AOA approaches $90 \mathrm{deg}$ at high airspeed $V . T_{\text {wing }}$ was found to rise with AOA and $V$. Also, the slope $\left(\partial T_{\text {wing }} / \partial \alpha_{p}\right)$ is increased with $V$ and $\left(\partial T_{\text {wing }} / \partial V\right)$ is increased with AOA, for a given $R P M$.

The theory shows that $T_{\text {wing }}$ surpasses $T_{\text {axial }}$ at $\mathrm{AOA} \approx 60 \mathrm{deg}$ or above and at high speeds $(w / V<0.6)$. Also, as the theoretical ratio blade configurations should present different surfaces of $T$ vs $w / V$ and AOA. Therefore, different slope sensitivities $\left(\partial T_{\text {wing }} / \partial V\right)$, $\left(\partial T_{\text {axial }} / \partial V\right)$ and $\left[\partial / \partial V\left(\partial T_{\text {wing }} / \partial \alpha_{p}\right)\right]$ should be expected, for a given RPM. It is believed that this could explain the inversion of $\partial T / \partial V$ at different angles of incidence for different propellers seen in other studies. However, the ratio $T_{\text {wing }} / T_{\text {axial }}$ must follow the theory, and the inversion of thrust behavior should happen around the region where $T_{\text {wing }}$ becomes relevant and ( $\left.\partial T_{\text {wing }} / \partial V\right)$ overcomes $\left(\partial T_{\text {axial }} / \partial V\right)$, which happens at high angles and at high speeds $(w / V<0.6)$. This verification could be done in further work.

At $\mathrm{AOA} \rightarrow 90 \mathrm{deg}, T_{\text {axial }}$ converges to the static thrust formula, being always present in the thrust composition even in forward flight. At hovering condition, when $T_{\text {wing }}$ vanishes, then $T_{\text {axial }}$ becomes the sole contributor of $T$, in which case the theory is proven to be consistent with the traditional formula $T=2 \rho S_{\text {disk }} w^{2}$. The proposed theory is also shown to agree completely with the classic momentum theory at $\mathrm{AOA}=0 \mathrm{deg}$. To a great extent, the increase of $T$ with AOA is due to the wing component. $T_{\text {wing }}$ peaks to compose most of the thrust at very high speed and high AOA. In this case, the proposed theory is shown to be consistent with Glauert's hypothesis, reverting to the wing lift formula used for helicopter rotors in fast forward flight, $T \rightarrow 2 \rho V w S_{\text {disk }}$.

The development presents an alternative simplified formula for estimating $T$ at any AOA up to $90 \mathrm{deg}$ for any propeller, based on data acquired at no incidence. ( $T$ or $C_{T}$ and $V, R P M$, or $J$ ). The formula showed good agreement with the experimental results for the propeller tested up to intermediate advance ratios and $\mathrm{AOA} \approx 80 \mathrm{deg}$.

A concluding remark is that one could assume the thrust of a propeller at incidence to be interpreted as the thrust of a propeller in axial flow condition under incoming speed of $V \cos \alpha_{p}$ with a thrust addition equivalent to the lift produced by an elliptic wing of area $S_{\text {disk }} W F$, under incoming speed $V \sin \alpha_{p}$, where both components share a common induced speed $w$.

\section{Appendix A: General Thrust Formula for Propellers at Incidence Based on Thrust at $\mathrm{AOA}=0 \mathrm{deg}$}

A final equation for thrust prediction based on propellers data at $\mathrm{AOA}=0 \mathrm{deg}$ is presented based on the assumption of $T_{\text {axial }}$ relatively constant with AOA up to around $60 \mathrm{deg}$ for intermediate $J$ values. The simplified equation is an approximation based on Eq. (39),

$$
\frac{T}{T_{\mid A=0 \text { deg }}}=\left\{1+\frac{\left(\sin \alpha_{p}\right)^{2}}{\left[\cos \alpha_{p}+\left(\frac{w}{V}\right)\right]\left[\sqrt{1+2\left(\frac{w}{V}\right) \cos \alpha_{p}+\left(\frac{w}{V}\right)^{2}}+\cos \alpha_{p}+\left(\frac{w}{V}\right)\right]}\right\}
$$

$T_{\text {wing }} / T_{\text {axial }}$ stems from the momentum theory development, which disregards blade geometry and propeller design, the authors assume that it would be valid for any propeller. The influence of $T_{\text {wing }}$ on $T$ overcomes that of $T_{\text {axial }}$ at high angles and speed as in those conditions the positive sensitivity $\left(\partial T_{\text {wing }} / \partial V\right)$ is larger than the negative sensitivity value of $\left(\partial T_{\text {axial }} / \partial V\right)$.

Thrust is found to be decreased with $V$ (and $J)(\partial T / \partial V<0)$ at low angles, as $T_{\text {axial }}$ is dominant, whereas at around $\mathrm{AOA} \approx 60 \mathrm{deg}$ or higher and at high airspeeds (and $J$ ), $T$ changes its behavior to increase with increasing $V$, in other words, $(\partial T / \partial V>0)$. This behavior is interpreted as being the consequence of $T_{\text {wing }}$ impact overcoming the contribution of $T_{\text {axial }}$ effects. Different propellers and where the value of $w / V$ is obtained from solving Eqs. (18) or (19), while using thrust data acquired at $\mathrm{AOA}=0 \mathrm{deg}$.

\section{Appendix B: Measured Different Thrust Behaviors for Tested AOA Range}

Constant AOA surfaces are depicted in Fig. B1, which shows $T$ as function of $V$ and RPM. It can be seen that below $\mathrm{AOA} \approx 60 \mathrm{deg}, T$ decreases with $V$, or $(\partial T / \partial V<0)$, for any given $R P M$, while for $\mathrm{AOA}>60 \mathrm{deg}, T$ grows with $V$, or $(\partial T / \partial V>0)$. This can be interpreted as the contribution of 


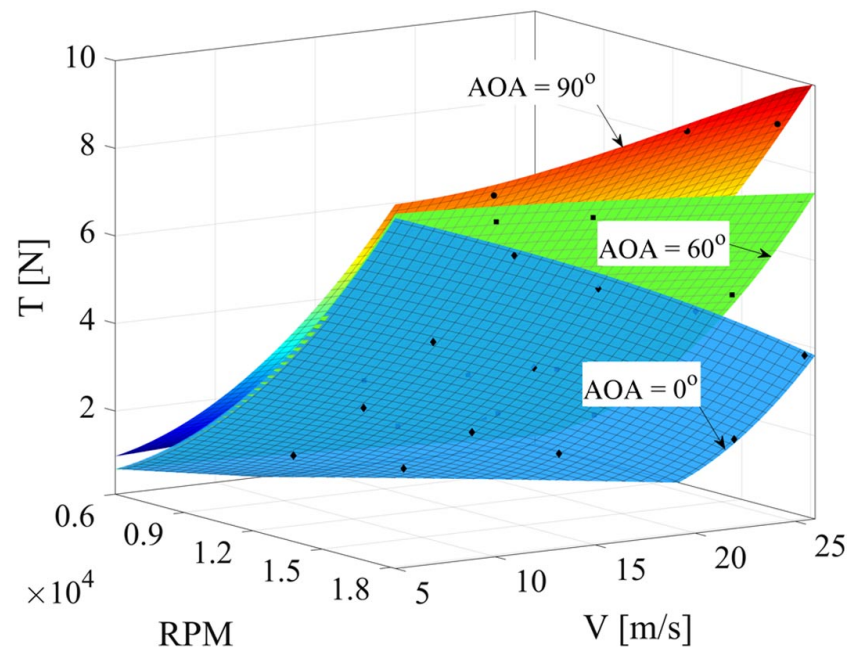

Fig. B1 Variation of the thrust $T$ with $V$ and $R P M$, as AOA is set to three different values.

$T_{\text {wing }}$ influence overcoming that of $T_{\text {axial }}$, in other words, $\left(\partial T_{\text {wing }} / \partial V>\partial T_{\text {axial }} / \partial V\right)$ at high angles, and vice versa at low angles.

\section{Acknowledgments}

The authors would like to express appreciation to the technical staff who helped make the experimental tests achievable, Julian Murphy, Julian Philips, Kenneth Brown, David Fanner, Natalia Kabaliuk, and Tony Doyle, and to Emeric Jego, an exchange engineering student from CESI University in France. The first author would like to thank Dan Zhao for supervising his Ph.D. research and this propeller work. The authors would also would like to thank the anonymous reviewers for their insightful and detailed comments.

\section{References}

[1] Rankine, W. M. J., "On the Mechanical Principles of the Action of Propellers," Transactions of the Institute of Naval Architects, Vol. 6, No. XX, 1865, pp. 13-39.

[2] Froude, R. E., "On the Part Played in Propulsion by Differences of Fluid Pressure," Transactions of the Institute of Naval Architects, Vol. 30, No. XX, 1889, pp. 390-405.

[3] Shapiro, J., Principles of Helicopter Engineering, McGraw-Hill, New York, 1956, pp. XX-XX.

[4] McCormick, B., Aerodynamics, Aeronautics \& Flight Mechanics, Wiley, New York, 1979, pp. XX-XX.

[5] McCormick, B. W., Jr., Aerodynamics of V/STOL Flight, Academic Press, New York, London, 1967, pp. XX-XX.

[6] Glauert, H., The Elements of Airfoil \& Airscrew Theory, Cambridge Univ. Press, Cambridge, England, U.K., 1948, pp. XX-XX.

\section{Appendix C: Experimental Measurement Error Analysis}

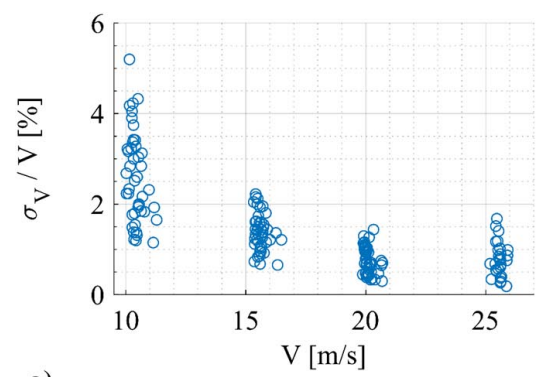

a)
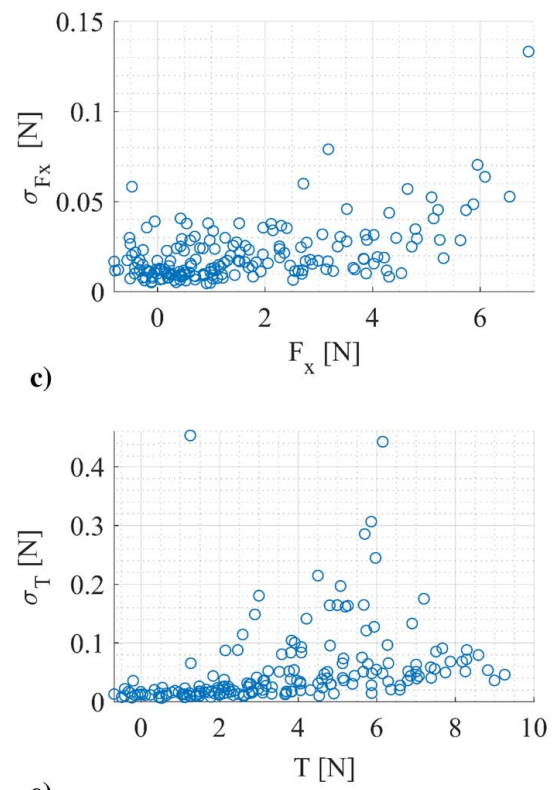

e)

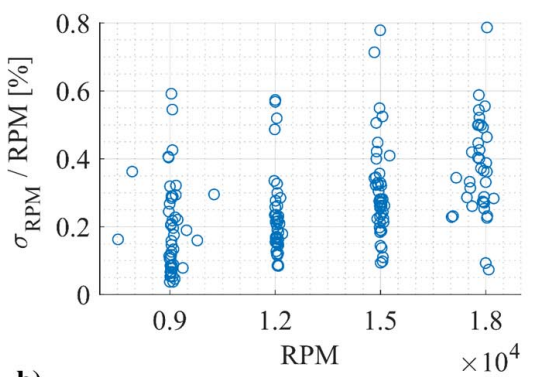

b)
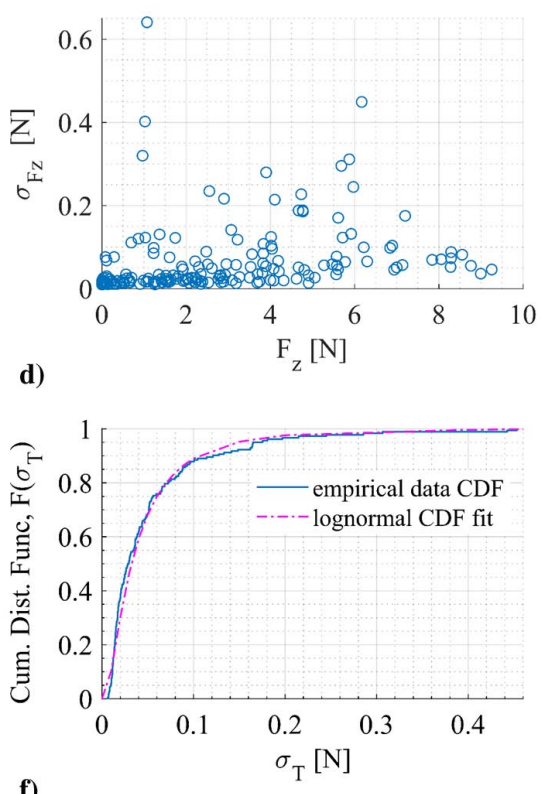

f)

Fig. C1 Measurement precision errors: a) in $V$, b) in $R P M$, c) in $F_{x}$, d) in $F_{z}$, e) inferred for $T$ from $F_{x}$ and $F_{z}$, and e) cumulative distribution function of the errors in $T$. 
[7] Glauert, H., "Airplane Propellers," Aerodynamic Theory-Vol. IV, edited byW. F. Durand, Springer-Verlag, Berlin, 1935, pp. 169-359.

[8] Dommasch, D. O., Elements of Propeller and Helicopter Aerodynamics, Pitman, London, 1953, pp. XX-XX.

[9] Betz, A., "Eine Erweiterung Der Schraubenstrahl Theorie," Zeitschrift für Flugtechnik und Motorluftschiffahrt, Vol. 11, No. XX, 1920, pp. 105-XXX

[10] Drzewiecki, S., Théorie Générale de l'hélice: Hélices Aériennes et Hélices Marines, Gauthier-Villars, XXXX, XX, 1920, pp. XX-XX

[11] Johnson, W., Helicopter Theory, Princeton Univ. Press, Princeton, NJ, 1980, pp. XX-XX.

[12] Goldstein, S., John, S., and Wilhelm, K., "On the Vortex Theory of Screw Propellers," Mathematical and Physical Character, Vol. 123, No. 792, 1929, pp. 440-465.

[13] Theodorsen, T., Theory of Propellers, McGraw-Hill, New York, 1948, pp. XX-XX.

[14] Van Kuik, G. A. M., Sørensen, J. N., and Okulov, V. L., "Rotor Theories by Professor Joukowsky: Momentum Theories," Progress in Aerospace Sciences, Vol. 73, Feb. 2015, pp. 1-18. https://doi.org/10.1016/j.paerosci.2014.10.001

[15] Okulov, V. L., Sørensen, J. N., and Wood, D. H., "The Rotor Theories by Professor Joukowsky: Vortex Theories," Progress in Aerospace Sciences, Vol. 73, Feb. 2015, pp. 19-46. https://doi.org/10.1016/j.paerosci.2014.10.002

[16] Ribner, H. S., "Propellers in Yaw," NACA Rept. 820, 1943.

[17] Ribner, H. S., "Formulas for Propellers in Yaw and Charts of the SideForce Derivative," NACA Rept. 819, 1943.

[18] De Young, J., "Propeller at High Incidence," Journal of Aircraft, Vol. 2, No. 3, 1965 , pp. $241-250$. https://doi.org/10.2514/3.43646

[19] McLemore, H. C., and Cannon, M. D., "Aerodynamic Investigation of a Four-Blade Propeller Operating Through an Angle-of-Attack Range From $0^{\circ}$ to $180^{\circ}, "$ NACA TN-3228, 1954.

[20] Kuhn, R. E., and Draper, J. W., "Investigation of the Aerodynamic Characteristics of a Model Wing-Propeller Combination and of the Wing and Propeller Separately at Angles of Attack up to $90^{\circ}$," NACA Rept. 1263, 1956.

[21] Yaggy, P. F., and Rogallo, V. L., "A Wind-Tunnel Investigation of Three Propellers Through an Angle- of-Attack Range from $0^{\circ}$ to $85^{\circ}$, , NACA TN D 318, 1960

[22] Ariza, D. G., "Study of the Sensitivity to the Lateral Wind of a Mini Unmanned Aerial Vehicle with VTOL Flight Capabilities," Ph.D. Dissertation, Inst. Supérieur de l'Aéronautique et de l'Espace, Toulouse, France, 2013.

[23] Serrano, D., Ren, M., Qureshi, A. J., and Ghaemi, S., "Effect of Disk Angle-of-Attack on Aerodynamic Performance of Small Propellers," Aerospace Science and Technology, Vol. 92, Sept. 2019, pp. 901914. https://doi.org/10.1016/j.ast.2019.07.022

[24] Øye, S., "Induced Velocities for Rotors in Yaw," Proceedings of the Sixth IEA Symposium, ECN, Petten, 1992, pp. XX-XX

[25] Haans, W., "Wind Turbine Aerodynamics in Yaw: Unravelling the Measured Rotor Wake," Ph.D. Dissertation, Delft Univ. of Technology, Delft, The Netherlands, 2011.

[26] Micallef, D., Sant, T., Aissaoui, A. G., and Tahour, A., "A Review of Wind Turbine Yaw Aerodynamics," Wind Turbines-Design, Control and Applications, edited by IntechOpen, IntechOpen, XXXX, XX, XXXX, pp. XX-XX.

[27] Rahimi, H., Hartvelt, M., Peinke, J., and Schepers, J. G., "Investigation of the Current Yaw Engineering Models for Simulation of Wind Turbines in BEM and Comparison with CFD and Experiment," Journal of Physics: Conference Series, Vol. 753, Sept. 2016, Paper 022016. https://doi.org/10.1088/1742-6596/753/2/022016

[28] Schepers, J., "An Engineering Model for Yawed Conditions, Developed on Basis of Wind Tunnel Measurements," 37th Aerospace Sciences Meeting and Exhibit, AIAA Paper 1998-0039, 1998. https://doi.org/10.2514/6.1999-39

[29] Schepers, J. G., "Engineering Models in Wind Energy Aerodynamics: Development, Implementation and Analysis Using Dedicated Aerodynamic Measurements," Ph.D. Dissertation, Delft Univ. of Technology, Delft, The Netherlands, 2012.

[30] Rahimi, H., Garcia, A. M., Stoevesandt, B., Peinke, J., and Schepers, G., "An Engineering Model for Wind Turbines Under Yawed Conditions Derived from High Fidelity Models," Wind Energy, Vol. 21, No. 8, 2018, pp. 618-633. https://doi.org/10.1002/we.2182

[31] Lin, M., and Porté-Agel, F., "Large-Eddy Simulation of Yawed Wind-Turbine Wakes: Comparisons with Wind Tunnel Measurements and Analytical Wake Models," Energies, Vol. 12, No. 23, 2019, pp. 4574-XXXX

https://doi.org/10.3390/en12234574

[32] Glauert, H., On the Horizontal Flight of a Helicopter, Her Majesty's Stationery Office, 1928, pp. XX-XX.

[33] Bramwell, A. R. S., "Some Remarks on the Induced Velocity Field of a Lifting Rotor and on Glauert's Formula," Aeronautical Research Council Current Papers-C.P. No.1301, Her Majesty's Stationery Office, 1974, pp. XX-XX

[34] Van Kuik, G. A. M., "On the Revision of the Actuator Disc Momentum Theory," Wind Engineering, Vol. 5, Jan. 1991, pp. 276-289.

[35] Van Kuik, G. A. M., "The Edge Singularity of an Actuator Disc with a Constant Normal Load," Proceedings of ASME Wind Energy Symposium, XXXX, XXXX, XX, 2003. https://doi.org/10.1115/wind2003-356

[36] Goorjian, P. M., "An Invalid Equation in the General Momentum Theory of Actuator Disk," AIAA Journal, Vol. 10, No. 4, 1972, pp. 543-544. https://doi.org/10.2514/3.50146

[37] Sorensen, J. N., and Mikkelsen, R., "On the Validity of the Blade Element Momentum Method," EWEC-CONFERENCE, XXXX, XXXX, XX, 2001, pp. 362-366.

[38] Van Kuik, G. A. M., and Lignarolo, L. E. M., "Potential Flow Solutions for Energy Extracting Actuator Disc Flows," Wind Energy, Vol. 19, No. 8, 2016, pp. 1391-1406. https://doi.org/10.1002/we.1902

[39] Bontempo, R., and Manna, M., "Verification of the Axial Momentum Theory for Propellers with a Uniform Load Distribution," International Journal of Turbomachinery, Propulsion and Power, Vol. 4, No. 2, 2019, pp. 8-X. https://doi.org/10.3390/ijtpp4020008

[40] Sorensen, J. N., General Momentum Theory for Horizontal Axis Wind Turbines, Springer-Verlag, XXXX, XX, 2016, pp. XX-XX.

[41] Bontempo, R., and Manna, M., "Analysis and Evaluation of the Momentum Theory Errors as Applied to Propellers," AIAA Journal, Vol. 54, No. 12,2016 , pp. 3840-3848. https://doi.org/10.2514/1.J055131

[42] Bontempo, R., and Manna, M., "Effects of the Approximations Embodied in the Momentum Theory as Applied to the NREL PHASE VI Wind Turbine," International Journal of Turbomachinery, Propulsion and Power, Vol. 2, No. 2, 2017, pp. 9-X. https://doi.org/10.3390/ijtpp2020009

[43] Conway, J. T., "Analytical Solutions for the Actuator Disk with Variable Radial Distribution of Load," Journal of Fluid Mechanics, Vol. 297, Aug. 1995, pp. 327-355. https://doi.org/10.1017/S0022112095003120

[44] Conway, J. T., "Exact Actuator Disk Solutions for Non-Uniform Heavy Loading and Slipstream Contraction," Journal of Fluid Mechanics, Vol. 365, June 1998, pp. 235-267. https://doi.org/10.1017/S0022112098001372

[45] Pitt, D. M., and Peters, D. A., "Theoretical Prediction of DynamicInflow Derivatives," Vertica, Vol. 5, No. XX, 1981, pp. 21-34.

[46] Morillo, J. A., and Peters, D. A., "Velocity Field Above a Rotor Disk by a New Dynamic Inflow Model,” Journal of Aircraft, Vol. 39, No. 5, 2002, pp. 731-738. https://doi.org/10.2514/2.3019

[47] Rosen, A., and Gur, O., "Novel Approach to Axisymmetric Actuator Disk Modeling," AIAA Journal, Vol. 46, No. 11, 2008, pp. 2914 2925.

https://doi.org/10.2514/1.37383

[48] Kominer, S., and Rosen, A., "New Actuator-Disk Model for a Skewed Flow," AIAA Journal, Vol. 51, No. 6, 2013, pp. 1382-1393. https://doi.org/10.2514/1.J052057

[49] Patterson, M. D., "Conceptual Design of High-Lift Propeller Systems for Small Electric Aircraft," Ph.D. Dissertation, Georgia Inst. of Technology, XXXX, GA, 2016.

[50] Veldhuis, L. L. M., "Propeller Wing Aerodynamic Interference," Ph.D. Dissertation, Delft Univ. of Technology, Delft, The Netherlands, 2005.

[51] Van Holten, T., "Concentrator Systems for Wind Energy, with Emphasis on Tipvanes," Wind Engineering, Vol. 5, No. 1, 1981, pp. 29-45.

[52] Dommasch, D. O., Sherby, S. S., and Connoly, T. F., Airplane Aerodynamics, Pitman Aeronautical, New York, 1951, pp. XX-XX.

[53] Deters, R. W., Ananda, G. K., and Selig, M. S., "Reynolds Number Effects on the Performance of Small-Scale Propellers," 32nd AIAA Applied Aerodynamics Conference, AIAA Paper 2014-2151, June 2014, pp. $1-43$. https://doi.org/10.2514/6.2014-215

[54] Ananda, G. K., Selig, M. S., and Deters, R. W., "Experiments of Propeller-Induced Flow Effects on a Low-Reynolds-Number Wing," 
AIAA Journal, Vol. 56, No. 8, 2018, pp. 3279-3294.

https://doi.org/10.2514/1.J056667

[55] Hrishikeshavan, V., and Chopra, I., "Performance, Flight Testing of a Shrouded Rotor Micro Air Vehicle in Edgewise Gusts," Journal of Aircraft, Vol. 49, No. 1, 2012, pp. 193-205.

https://doi.org/10.2514/1.C031477
[56] Brandt, J. B., Deters, R. W., Ananda, G. K., and Selig, M. S., UIUC Propeller Database, University of Illinois at Urbana-Champaign, http:// m-selig.ae.illinois.edu/props/propDB.html [retrieved 22 April 2020].

F. N. Coton Associate Editor 


\section{Queries}

1. AU: To make sure the author footnote for Rubin is styled correctly, please choose from one of the following approaches: A) It is customary to list the affiliation at the time of writing at the top, and a subsequent affiliation can be listed in the footnote. For this approach, please indicate which affiliation was at the time of writing and which is current. B) If preferred, only the current affiliation can be listed at the top. For this approach, please indicate the former affiliation and it will be removed. C) If the author currently has a double affiliation, please indicate which one is the primary and should appear at the top. Please also note that APS only lists the highest job title for any given affiliation, so if Ph.D. Candidate and Aerospace Engineer are both for the Univ. of Canterbury affiliation, please specify which is highest.

2. AU: Please check changes to the sentence "While thrust decreases ..."

3. AU: Please provide units of measure for all nomenclature entries for which a unit of measure applies.

4. AU: Please check added definitions of VTOL and UAV.

5. AU: To what does "it" refer in the sentence "It was first...?"

6. AU: Please check changes to the sentence "Extensive integrations of ..."

7. AU: The sentence "This is due..." is unclear. Please reread the sentence.

8. AU: What is the significance of the parentheses around the math in the sentence "As the airflow ...?"

9. AU: The sentence containing Eq. (8) is unclear. Please rewrite the sentence.

10. AU: Journal style forbids starting a sentence with a lowercase variable. Please rewrite the sentence after Eq. (24) to avoid this.

11. TE: Please change the degree symbol in the math throughout to "deg".

12. AU: Please check changes to the sentence "The force balance..."

13. AU: Please define HQ.

14. AU: Please check changes to the sentence "The propeller used ..."

15. AU: The sentence "Presenting the forces ..." is unclear. Please rewrite.

16. AU: the sentence "The maximum error..." is unclear. Please rewrite.

17. AU: Please check RPM changed to rpm when it is used as a unit of measure, which was done to better fit journal style.

18. AU: The sentence "At $\mathrm{AOA}=0 \ldots$... is unclear. Please rewrite.

19. AU: To whom or what does "they" refer in the sentence "They found the ...?"

20. AU: Please check changes to the sentence "A possible explanation..."

21. AU: What do you mean by "where $T \rightarrow T_{\text {wing }}$ formula according to?" 
22. AU: Please define CDF in Fig. C1.

23. AU: Please spell out CESI.

24. AU: Please provide the issue number and/or month of publication for Refs. [1, 2, 45] if applicable.

25. AU: For Refs. [3, 5,6,8, 11, 13, 32, 52], please provide page range or chapter number.

26. AU: For Refs. [4, 10, 40], please provide publisher location and page range or chapter number.

27. AU: Please provide the ending page number for Ref. [9].

28. AU: In Ref. [24], please spell out ECN and provide the state or country where Petten is located.

29. AU: If citing specific pages from Refs. [24, 33], please provide them.

30. AU: For Ref. [26], please provide publisher location, date of publication, and page range. Double check the name of the editor.

31. AU: Please provide the ending page number for Ref. [31]?

32. AU: If Ref. [35] is a published proceedings, please provide the full name and location of the publisher (NOT of the conference host) and the page range. If it is a conference paper, please provide the paper number and the organizer's name.

33. AU: For Ref. [37], please provide publisher name and location.

34. AU: Please provide the ending page number for Ref. [39, 42].

35. AU: Please provide the city in Georgia for Ref. [49]'s school.

\section{Funding Information}

No funding information available. 